\title{
Environmental Factors and Pollution Stresses Select Bacterial Populations in Association With Protists
}

\author{
Songbao Zou'1,2,3, Qianqian Zhang ${ }^{1}$, Xiaoli Zhang ${ }^{1}$, Christine Dupuy ${ }^{4}$ and Jun Gong ${ }^{3,5 *}$ \\ ${ }^{1}$ Yantai Institute of Coastal Zone Research, Chinese Academy of Sciences, Yantai, China, ${ }^{2}$ University of Chinese Academy \\ of Sciences, Beijing, China, ${ }^{3}$ School of Marine Sciences, Sun Yat-sen University, Zhuhai, China, ${ }^{4}$ Littoral Environnement et \\ Sociétés (LIENSs) UMR 7266 CNRS, University of La Rochelle, La Rochelle, France, ${ }^{5}$ Southern Marine Science \\ and Engineering Guangdong Laboratory (Zhuhai), Zhuhai, China
}

\section{OPEN ACCESS}

Edited by:

Hongbo Pan,

Shanghai Ocean University, China

Reviewed by:

Martina Schrallhammer,

University of Freiburg, Germany

Zhenzhen Yi,

South China Normal University, China

*Correspondence: Jun Gong

gongj27@mail.sysu.edu.cn

Specialty section This article was submitted to Marine Evolutionary Biology, Biogeography and Species Diversity, a section of the journal

Frontiers in Marine Science

Received: 15 June 2020

Accepted: 21 July 2020

Published: 07 August 2020

Citation:

Zou S, Zhang Q, Zhang X,

Dupuy C and Gong J (2020) Environmental Factors and Pollution Stresses Select Bacterial Populations

in Association With Protists.

Front. Mar. Sci. 7:659

doi: 10.3389/fmars.2020.00659
Digestion-resistant bacteria (DRB) refer to the ecological bacterial group that can be ingested, but not digested by protistan grazers, thus forming a specific type of bacteria-protist association. To test the hypothesis that the environment affects the assembly of DRB in protists, a mixotrophic ciliate, Paramecium bursaria, and a heterotrophic ciliate, Euplotes vannus, were reared at different temperatures, light conditions, and concentration gradients of antibiotic oxytetracycline and heavy metals. Community profiling indicated that the composition of DRB in both species varied significantly across the manipulated conditions, except for in $P$. bursaria under light/dark treatments. Clone library analysis of bacterial 16S rRNA genes showed that DRB were diverse. Pseudomonas became more abundant during the warmer treatment of $P$. bursaria, whereas the dominance of Pseudoalteromonas weakened and Vibrio became more abundant in E. vannus at a higher temperature. During the treatment of diel light:dark cycles, Aestuariibacter and Alteromonas were selected for in E. vannus but not Pseudoalteromonas, which was highly represented in the all-light and alldark treatments. In contrast, P. bursaria consistently hosted Nevskia, Curvibacter, and Asticcacaulis under all light conditions. There were many bacterial species co-resistant to oxytetracycline and to protistan digestion, in which Sphingomonas, Alteromonas, Aestuariibacter, Puniceicoccaceae (Verrucomicrobia), Pseudomonas, and Sulfitobacter were frequently abundant. Flectobacillus and Aestuariibacter were major lead-resistant bacteria associated with the studied protists. Acinetobacter and Hydrogenophaga were abundant in the $P$. bursaria treated with a high dose of mercury. Aestuariibacter was found as a dominant group of DRB in E. vannus across all cadmium treatments. In summary, this study demonstrates for the first time that environmental stress selects for bacterial populations associated with protists and that there are diverse bacterial species that not only are resistant to pollution stresses but can also survive protistan predation. This work highlights that bacteria-protists associations need to be taken into account in understanding ecological and environmental issues, such as resilience of bacterial community and function, microbial co-occurrence, and quantity and distribution of antibiotic resistant bacteria and genes.

Keywords: antibiotic resistance, interkingdom interaction, light, metal resistance, protozoa, warming 


\section{INTRODUCTION}

Bacteria and protists are major players in the microbial food web of aquatic environments, in which heterotrophic bacteria incorporate dissolved organic matter and nutrients for growth and transfer carbon and energy to higher trophic levels as a consequence of predation by small protists (Azam et al., 1983). In the last two decades, much efforts have been made to understand how and why protists selectively graze on bacteria or how bacteria become resistant to protistan predation. It has been demonstrated that changes in bacterial cell size, morphology, movement, cellcell configuration in space, and chemical resistance contribute to their anti-predation (Matz and Kjelleberg, 2005; Pernthaler, 2005; Jousset, 2012). Furthermore, recent studies have provided evidence for another strategy of anti-predation-some bacterial populations in aquatic environments can be ingested, but not digested by protistan grazers, thus potentially forming microbial associations with the protistan hosts (e.g., First et al., 2012; Šimek et al., 2013; Gong et al., 2014). When protistan isolates and assemblages are exposed in natural bacterial communities, the detected digestion-resistant bacteria (DRB) can be prevalent and diverse, showing a common trait in taxonomic composition where Gammaproteobacteria and Alphaproteobacteria usually dominate in marine samples (Martinez-Garcia et al., 2012; Pucciarelli et al., 2015; Farnelid et al., 2016; Gong et al., 2016). From an ecological point of view, the existence of DRB could prevent organic matter and energy from being transferred to protistan grazers and higher trophic levels via the microbial loop, adding another layer of complexity in the structure and functions of microbial webs.

Despite the ecological importance of DRB, associations between DRB and protists remain poorly understood. One issue is how environment and host affect the diversity and assemblage composition of the DRB associated with protistan populations. There are indications that pre-culture conditions would affect the ingestion and digestion of heterotrophic nanoflagellates (Boenigk et al., 2001). In our previous studies of DRB in ciliated protists cultivated in the laboratory or isolated directly from natural environments, we also observed that the DRB assemblage could substantially differ among host species and even for populations of the same species isolated at separate time points at an identical location, suggesting that the DRB diversity and assemblage composition can be both host- and environmentdependent (Gong et al., 2014, 2016). These relationships seem straightforward, since numerous studies have demonstrated that changes in physical and chemical factors drive bacterial abundance and community composition in bulk environmental samples via bottom-up controls or due to effects of pollutants (e.g., Storesund et al., 2015; Ibekwe et al., 2016).

It is well known that environmental factors play important roles in shaping the diversity and dynamic of the bacteria in a natural aquatic environment (Alonso-Sáez et al., 2006; Adams et al., 2010; Darriba et al., 2012; Hu et al., 2014). When the diversity of source bacteria shifts via environmental filtering, it is likely that different sets of bacterial populations are ingested by the protists and retained inside the cells for at least a short-term period, resulting in different assemblage structures of
DRB accordingly. However, when the ingested bacteria escape from lysosomes, they enter into the cytoplasm of host cells, a relatively stable physical and chemical environment (due to cellular homeostasis), which may select for a specific group of DRB. However, it remains to be investigated if and how DRB assemblage associated with bacterivorous protists varies with environmental changes and under chemical stresses.

In this study, we performed microcosm experiments using two bacterivorous protist species, Euplotes vannus, a marine heterotrophic ciliate, and Paramecium bursaria, a freshwater ciliate with endosymbiotic green algae (Chlorella sp.). We aimed to test the following hypotheses: (1) warming, light condition, antibiotic, and heavy metal treatments would individually lead to significant changes in DRB assemblages of both ciliate species; (2) the changes in DRB assemblages in both ciliates were greater than the free-living bacterioplankton in the media; and (3) there were diverse bacteria co-resistant to antibiotics/metals and protistan digestion. We believe that the exploration of the diversity and variation pattern of DRB will improve our understanding of bacteria-protist interactions and the dynamics of microbial food webs.

\section{MATERIALS AND METHODS}

\section{Source Organisms and Cultivation}

The freshwater ciliate species $P$. bursaria, collected from a pond on the campus of Yantai University (Yantai, Shandong, China) in 2014, and the marine species E. vannus, isolated from the estuarine water of the Guangdang River (Yantai, China) in 2013, were investigated. The water used for cultivation was collected on site and treated by passing through a GF/C glass fiber filter (1822-047 grade, pore size: $1.2 \mu \mathrm{m}$, Whatman, United Kingdom), such that most pico-, nano-, and larger-sized organisms were removed, and the raw bacterioplankton was largely retained as food for the testing protists. The ciliate cells were observed under a stereoscope (XLT-400, Guilin Guiguang, Guangxi, China), individually selected with a micropipette, and washed for at least 3 times in sterile water to remove other protists and attaching bacteria. A few (3 to 5) cleaned cells were then inoculated into filtered water, allowing the stock cultures to develop. The stock cultures (each in $15 \mathrm{~mL}$ volume) were maintained in glass Petri dishes at $16^{\circ} \mathrm{C}$ for 2 weeks, with several rice grains to enrich bacterial prey (Supplementary Figure S1).

\section{Experimental Settings}

Different temperatures, illuminations, and stresses of antibiotics and heavy metals were set up to investigate the effects of environmental factors and pollution stresses on the diversity of DRB. For each treatment, about 30 ciliate individuals were picked up from the stock cultures and transferred into a Petri dish containing $20 \mathrm{ml}$ aliquote of the filtered water, in which we assumed there was a bacterial community initially identical in quantity and community composition but subsequently being selectively grazed under various treatment conditions (Supplementary Figure S1). Effects of each factor were investigated independently, and each treatment was performed in 
triplicate. To investigate the effect of temperature, the Petri dishes with stock ciliate cultures were incubated in three chemostats, which were set at a temperature of $16^{\circ} \mathrm{C}, 21^{\circ} \mathrm{C}$, and $25^{\circ} \mathrm{C}$, with other factors kept constant (e.g., with $12 \mathrm{~h}: 12 \mathrm{~h}$ alternation of light and dark and good gas exchange). The experiments testing the effects of light and pollutant stresses were performed at a constant temperature of $25^{\circ} \mathrm{C}$. Light treatments were maintained in consistently illuminated (LL), completely dark (DD), and $12 \mathrm{~h}: 12 \mathrm{~h}$ alternation of light and dark conditions (LD).

The effects of pollution stresses on the DRB assemblage were carried out along gradients of the pollutants. To examine the effects of antibiotics, oxytetracycline, one of the most widely used antibiotics in farming industrials, was used in this study. The stock solution (100 mg/L) of oxytetracycline (OTC, Sigma, St. Louis, MO, United States) was added, drop-by-drop, to the ciliate cultures and gently mixed, resulting in final concentrations of 1 , 10 , and $20 \mathrm{mg} / \mathrm{L}$ (hereafter referred to as OTC1, OTC10, and OTC20, respectively). Three types of heavy metals, lead chloride $\left(\mathrm{PbCl}_{2}\right)$, cadmium chloride $\left(\mathrm{CdCl}_{2}\right)$, and mercury chloride $\left(\mathrm{HgCl}_{2} ; \mathrm{AR}\right.$ grade; Sinopharm, Shanghai, China) were used at final concentrations as follows: 10,30 , and $50 \mathrm{mg} / \mathrm{L}$ for $\mathrm{PbCl}_{2}$ (hereafter referred to as $\mathrm{Pb} 10, \mathrm{~Pb} 30$, and $\mathrm{Pb} 50$ ); 0.01, 0.05, and $0.1 \mathrm{mg} / \mathrm{L}$ for $\mathrm{HgCl}_{2}$ (hereafter referred to as $\mathrm{Hg} 0.01, \mathrm{Hg} 0.05$, and $\mathrm{Hg} 0.1$ ); and $0.1,0.5$, and $1 \mathrm{mg} / \mathrm{L}$ for $\mathrm{CdCl}_{2}$ (hereafter referred to as $\mathrm{Cd} 0.1, \mathrm{Cd} 0.5$, and Cd1). Since neither P. bursaria in the $\mathrm{Cd}$ treatments nor $\mathrm{E}$. vannus in the $\mathrm{Hg}$ treatments survived, these treatments were not included in the subsequent analyses. The cultures without any amendments of antibiotic and heavy metals (i.e., the treatments OTC0, $\mathrm{Pb} 0, \mathrm{Hg} 0$, and $\mathrm{Cd} 0$ ) were taken as the controls.

For molecular assays of DRB inside the protistan cells, approximately 20-30 ciliate cells in all of these treatments were isolated during their exponential growth stages, which usually took place within 8 days of cultivation according to our pilot studies. In order to minimize the contamination from the bacteria attached to the cell surface and the cilia, protistan cells from these treatments were washed repeatedly (at least three times) by transferring to a series of sterilized seawater (for E. vannus) or autoclaved Milli-Q water (for P. bursaria) with a clean micropipette. These cleaned ciliates were then maintained in the sterilized water for two or three days, allowing the ingested bacteria to be digested as much as possible. The remaining bacteria inside these ciliate cells were considered DRB. Since the free-living bacteria in the culture medium were apparently sources for the DRB, the diversity of the former might be altered under different conditions, which was a potential mechanism modulating the diversity of the latter. To investigate this, therefore the ciliate-free culture medium was also collected for analyzing the free-living bacterial community composition and structure in each treatment.

\section{DNA Extraction, Clone Libraries, and Sequencing}

Three to five ciliate individuals with a minimum volume of water were transferred to a microfuge tube for DNA extraction using a REDExtract-N-Amp Tissue PCR Kit (Sigma, St. Louis,
MO, United States), according to the modified protocol (Gong et al., 2014). Furthermore, the DNA of bacterioplankton was prepared by taking $3 \mu \mathrm{L}$ of ciliate-free culture medium from each treatment using the same method.

The identities of the DRB were revealed using PCR amplification, clone library construction, and sequencing of bacterial 16S rRNA genes. PCR was carried out in a Applied Biosystems Veriti $^{\circledR}$ 96-Well thermal cycler (Foster City, CA, United States), with universal bacterial 16S rRNA gene primers 27F1 (5'-AGAGTTTGATCCTGGCTCAG-3') and 1492R $\left(5^{\prime}\right.$-GGTTACCTTGTTACGACTT-3') (Lane, 1991), or $63 \mathrm{~F}$ $\left(5^{\prime}\right.$-CAGGCCTAACACATGCAAGTC- $\left.3^{\prime}\right)$ and 1389R (5'ACGGGCGGTGTGTACAAG-3') (Osborn et al., 2000). Each PCR reaction cocktail contained $1 \mu \mathrm{L}$ of $10 \mu \mathrm{M}$ forward and reverse primers, $1 \mu \mathrm{L}$ of template DNA, $2.5 \mu \mathrm{L}$ of $10 \times$ PCR buffer, $2.5 \mu \mathrm{L}$ of $25 \mathrm{mmol} / \mu \mathrm{L} \mathrm{MgCl}_{2}, 0.5 \mu \mathrm{L}$ of $10 \mathrm{mmol} / \mu \mathrm{L}$ dNTP, and $0.2 \mu \mathrm{L}$ of $1 \mathrm{U}$ Taq DNA polymerase (Fermentas, Thermo Scientific, Waltham, MA, United States), and deionized water was added to a final volume of $25 \mu \mathrm{L}$. The PCR reactions were programmed as follows: an initial denaturation step at $94^{\circ} \mathrm{C}$ for $3 \mathrm{~min}$, followed by 35 cycles of $30 \mathrm{~s}$ denaturation at $94^{\circ} \mathrm{C}, 1 \mathrm{~min}$ annealing (primers $27 \mathrm{~F} / 1492 \mathrm{R}$ at $50^{\circ} \mathrm{C}$, and primers $63 \mathrm{~F} / 1389 \mathrm{R}$ at $52^{\circ} \mathrm{C}$ ), and a $2 \mathrm{~min}$ elongation at $72^{\circ} \mathrm{C}$, with a final extension step of $72^{\circ} \mathrm{C}$ for $7 \mathrm{~min}$. Subsequently, all PCR products were visualized on $1 \%$ agarose gels, which contained GoldView (Solarbio, Beijing, China). The target bands were excised, purified by a TIANquick Midi Purification Kit (Tiangen, Beijing, China), inserted into PTZ57 R/T vectors using the InsTAclone PCR Clone Kit (Fermentas), and transferred to the competent cells Trans $5 \alpha$ (TransGen Biotech, Beijing, China) in order to construct the clone libraries. A total of 108 clone libraries were constructed, with about 50 positive clones randomly selected for pre-screening, which was conducted using restriction fragment length polymorphism (RFLP) analysis with Taq and HhaI, two FastDigest restriction enzymes (Thermo Scientific, Waltham, MA, United States). About 20-30 clones in each library were sequenced on an automated ABI 373 DNA sequencer (Applied Biosystems) in a commercial sequencing service company (Sangon Biotech, Shanghai, China).

The 16S rRNA gene sequences (about 1300 bp in length) obtained from cloning and sequencing were aligned using MAFFT v.7.0 with default parameters (Katoh and Standley, 2013). Potential chimeras were identified using Bellerophon (Huber et al., 2004) and Mallard 1.02 (Ashelford et al., 2006) and discarded before subsequent analysis. The cleaned sequences were clustered into operational taxonomic units (OTUs) at 97\% identity using Mothur v.1.17 (Schloss et al., 2009). Taxonomic identities and systematic placements of the OTUs were assigned using the RDP Classifier (release 11.5) (Wang et al., 2007). The $16 \mathrm{~S}$ rRNA gene sequences have been deposited in the GenBank databases with accession numbers MH555909 to MH556816.

\section{Phylogenetic Analyses}

For several sequences that assigned into unclassified Bacteroidetes and Verrucomicrobia by the RDP classifier, phylogenetic analyses were carried out using both maximum 
likelihood (ML) and Bayesian inference (BI) algorithms to further resolve their taxonomic ranks. Reference sequences of previously described species were retrieved from the GenBank database and aligned with the sequences in question using MAFFT. The alignment was refined manually using SeaView4 (Galtier et al., 1996). The models of nucleotide substitution were estimated using jModeltest2 (Darriba et al., 2012) based on Akaike information criterion (AIC) and Bayesian information criterion (BIC). The ML analysis was conducted using PhyML v.3.3 (Guindon et al., 2009) with a best-fit GTR + I + G evolutionary model and 1000 bootstrap replicates. The BI trees were generated using MrBayes 3.2.7 with selected parameters ( rates $=$ invgamma, number of substitution types $=6$, ncat $=4$ ) corresponding to the best model selected (GTR $+\mathrm{I}+\mathrm{G})$ (Ronquist and Huelsenbeck, 2003), implemented using Markov Chain Monte Carlo simulations and four chains running for 3,000,000 generations. The first $25 \%$ of generations were discarded as burn-in.

\section{Profiling of Bacterial Assemblages}

Variations in the assemblage structure of both the DRB associated with the protists and the bacterioplankton in the culture medium (but outside the ciliate cells) among the treatments were assessed using terminal restriction fragment length polymorphism (TRFLP). Bacterial 16S rRNA genes were amplified as described above, except that the forward primer $63 \mathrm{~F}$ was labeled with a fluorescent dye, 6-carboxyfluorescein, at its $5^{\prime}$ end. The PCR products were purified with the TIAN Quick Midi Purification Kit (Tiangen, Beijing, China). The DNA yield was quantified using a NanoDrop 2000C spectrophotometer (Thermo Fisher, Wilmington, DE, United States). A $15 \mu \mathrm{L}$ digestion mixture containing $7.5 \mu \mathrm{L}$ of purified PCR product (each with about $20 \mathrm{ng}$ of DNA) and $7.5 \mu \mathrm{L}$ of digestion buffers with restriction endonuclease HhaI (Fermentas) were incubated at $37^{\circ} \mathrm{C}$ for $1 \mathrm{~h}$. Sizes of the terminal restriction fragments (T-RFs) were determined using an ABI 3730 DNA analyzer (Sangon Biotech, Shanghai, China). The baseline threshold for signal detection was set to 50 fluorescence intensity units to eliminate any background interference. Only the peaks with T-RF lengths ranging from 40 to $400 \mathrm{bp}$ were included in the subsequent analysis. The relative abundance of each T-RF was calculated as the peak area ratio of the T-RF to all T-RFs detected for a given sample. Minor peaks with a relative abundance of $<1 \%$ of the total were excluded, and the remaining peaks were presumed to represent bacterial phylotypes.

The assemblage information generated from the T-RFLP profiling was analyzed using the software package PRIMER v.6 (Primer-E, Plymouth, United Kingdom). In order to test the differences in DRB assemblages, relative abundances of T-RFs were log-transformed, and a matrix of assemblage similarities was generated using the Bray-Curtis index. Nonmetric multidimensional scaling (MDS) was executed to visualize the differences in DRB assemblage structure among the treatments of each factor. To test the hypotheses that a given factor would affect the assemblage structure of the DRB and the bacterioplankton in the waters, analysis of similarity (ANOSIM) was performed.

\section{RESULTS}

\section{Community Profiling of Bacterioplankton in the Culture Media}

The T-RFLP profiling and ANOSIM results of global tests showed that all environmental factors investigated in this study, i.e., temperature $(R \geq 0.71, P \leq 0.014)$, light $(R \geq 0.38, P \leq 0.039)$, OTC $(R \geq 0.47, P=0.001), \mathrm{Pb}(R \geq 0.75, P \leq 0.002), \mathrm{Hg}(R=1.00$, $P=0.001)$, and $\mathrm{Cd}(R=0.76, P=0.001)$, significantly affected the community structure of bacterioplankton in the culture media for the tested ciliate species (Table 1). From the point view of the $R$ statistic values of global tests, the effects of the tested heavy metals on the bacterioplankton community structure seem to be stronger than those of temperature, OTC antibiotics, and light.

\section{Community Profiling of DRB}

The DRB assemblages were clearly separated into three groups by temperature in E. vannus, which was well-illustrated in the MDS plots (Figure 1B), while the temperature-specific clusters somewhat overlapped in P. bursaria (Figure 1A). The analysis of the assemblage structure of the DRB generally showed similar results of the bacterioplankton, namely, the effects of temperature (global test, $R \geq 0.50, P=0.001)$, OTC $(R \geq 0.49, P=0.001)$, and $\mathrm{Pb}(R \geq 0.80, P=0.001)$ were significant for the DRB in both species. Testing on a single species also illustrated that $\mathrm{Hg}(R=0.73, P=0.001)$ was an influential factor in the assemblage structure of DRB in P. bursaria, and Cd for that in E. vannus $(R=0.79, P=0.001)$. The only exception was the light treatments in P. bursaria, in which the assemblage of DRB was not significantly different among the LL, LD, and DD treatments $(R=0.05, P=0.362)$ (Figure $1 \mathrm{C}$ and Table $\mathbf{1}$ ).

The MDS plots showed that, within the same range of a given environmental factor, the DRB assemblages were clearly separated into three groups in the marine species but overlapped somewhat in the freshwater form (Figure 1). These patterns were also supported by the results of ANOSIM: the $R$-value of the DRB assemblage was consistently lower than that of the bacterioplankton community in all treatments of $P$. bursaria (i.e., $\Delta R_{\text {mean }}=R_{\mathrm{DRB}}-R_{\text {water }}=-0.22 ; n=5$ ) but higher in those of E. vannus (i.e., $\Delta R_{\text {mean }}=R_{\mathrm{DRB}}-R_{\text {water }}=0.15 ; n=5$; Table 1). This indicated that the DRB assemblage was less variable in $P$. bursaria but more variable in E. vannus as compared with their surrounding bacterial prey under varying temperature, light, OTC, $\mathrm{Pb}, \mathrm{Hg}$, and $\mathrm{Cd}$ concentrations. The $\mathrm{DRB}$ and bacterioplankton community were also significantly different in a given treatment, especially under highly stressed conditions (e.g., at $25^{\circ} \mathrm{C}$ or in OTC20, see Supplementary Table S1).

\section{Identity and Classification of Digestion-Resistant Bacteria}

A total of 1018 raw sequences of 16S rRNA genes were obtained from 108 clone libraries and sequencing. After removing 110 chimeras, 908 remained (Figure 2 and Table 2). Overall, most of these DRB belonged to the phylum Proteobacteria (61.3\% for $P$. bursaria and $87.4 \%$ for $E$. vannus). The phylotypes belonging to Bacteroidetes (25.7\%), Actinobacteria (13.8\%), 
TABLE 1 | Analysis of similarities (ANOSIM) statistics shows the differences in the community structures of digestion-resistant bacteria (DRB) in ciliates Paramecium bursaria and Euplotes vannus, as well as the bacterioplankton in their culture medium subjected to environmental changes.

\begin{tabular}{|c|c|c|c|c|c|c|c|c|}
\hline \multirow[t]{3}{*}{ Comparison } & \multicolumn{4}{|c|}{ P. bursaria } & \multicolumn{4}{|c|}{ E. vannus } \\
\hline & \multicolumn{2}{|c|}{ Bacterioplankton } & \multicolumn{2}{|c|}{ DRB } & \multicolumn{2}{|c|}{ Bacterioplankton } & \multicolumn{2}{|c|}{ DRB } \\
\hline & $\boldsymbol{R}$ & $P$ & $\boldsymbol{R}$ & $P$ & $\boldsymbol{R}$ & $P$ & $\boldsymbol{R}$ & $P$ \\
\hline Temperature (global test) & 0.77 & 0.014 & 0.50 & 0.001 & 0.71 & 0.004 & 0.86 & 0.001 \\
\hline $16^{\circ} \mathrm{C}$ vs. $21^{\circ} \mathrm{C}$ & 0.04 & 0.400 & 0.15 & 0.071 & 0.96 & 0.100 & 0.44 & 0.008 \\
\hline $21^{\circ} \mathrm{C}$ vs. $25^{\circ} \mathrm{C}$ & 1.00 & 0.100 & 0.21 & 0.087 & 0.48 & 0.100 & 1.00 & 0.008 \\
\hline $16^{\circ} \mathrm{C}$ vs. $25^{\circ} \mathrm{C}$ & 1.00 & 0.100 & 0.11 & 0.127 & 0.93 & 0.100 & 0.88 & 0.008 \\
\hline Light (global test) & 0.41 & 0.014 & 0.05 & 0.362 & 0.38 & 0.039 & 0.87 & 0.001 \\
\hline LL vs. DD & 0.33 & 0.400 & 0.19 & 0.400 & -0.30 & 0.900 & 0.59 & 0.008 \\
\hline LL vs. LD & 0.56 & 0.100 & 0.33 & 0.300 & 0.89 & 0.100 & 0.96 & 0.008 \\
\hline DD vs. LD & 0.33 & 0.400 & 0.00 & 0.700 & 0.52 & 0.100 & 1.00 & 0.008 \\
\hline ОTC (global test) & 0.57 & 0.001 & 0.53 & 0.001 & 0.47 & 0.001 & 0.49 & 0.001 \\
\hline OTC0 vs. OTC1 & 0.56 & 0.100 & 0.12 & 0.214 & 0.11 & 0.300 & 0.07 & 0.214 \\
\hline OTC0 vs. OTC10 & 0.56 & 0.100 & 0.62 & 0.008 & 0.67 & 0.100 & 0.17 & 0.103 \\
\hline ОTCO vs. ОTC20 & 0.59 & 0.100 & 0.66 & 0.600 & 1.00 & 0.100 & 0.35 & 0.008 \\
\hline OTC1 vs. OTC10 & 0.93 & 0.100 & 0.05 & 0.706 & 0.30 & 0.100 & 0.51 & 0.016 \\
\hline OTC1 vs. OTC20 & 1.00 & 0.100 & 0.23 & 0.048 & 0.37 & 0.100 & 0.52 & 0.016 \\
\hline OTC10 vs. OTC20 & -0.15 & 0.100 & 0.20 & 0.103 & 0.63 & 0.100 & 0.32 & 0.016 \\
\hline $\mathrm{Pb}^{2+}$ (global test) & 1.00 & 0.001 & 0.83 & 0.001 & 0.75 & 0.002 & 0.80 & 0.001 \\
\hline $\mathrm{Pb0}$ vs. Pb10 & 1.00 & 0.100 & 0.63 & 0.029 & 0.93 & 0.100 & 0.24 & 0.086 \\
\hline $\mathrm{Pb0}$ vs. Pb30 & 1.00 & 0.100 & 0.38 & 0.029 & 0.56 & 0.100 & 0.84 & 0.005 \\
\hline $\mathrm{Pb0}$ vs. Pb50 & 1.00 & 0.100 & 0.56 & 0.029 & 1.00 & 0.100 & 0.46 & 0.005 \\
\hline Pb10 vs. Pb30 & 1.00 & 0.100 & 0.42 & 0.057 & 0.70 & 0.200 & 1.00 & 0.029 \\
\hline Pb10 vs. Pb50 & 1.00 & 0.100 & -0.01 & 0.486 & 0.59 & 0.100 & 0.40 & 0.057 \\
\hline Pb30 vs. Pb50 & 1.00 & 0.100 & 0.33 & 0.086 & 0.74 & 0.100 & 0.71 & 0.029 \\
\hline $\mathrm{Hg}^{2+}$ (global test) & 1.00 & 0.001 & 0.73 & 0.001 & - & - & - & - \\
\hline HgO vs. Hg0.01 & 1.00 & 0.100 & 0.25 & 0.029 & - & - & - & - \\
\hline HgO vs. Hg0.05 & 1.00 & 0.100 & 0.51 & 0.029 & - & - & - & - \\
\hline HgO vs. Hg0.1 & 1.00 & 0.100 & 0.09 & 0.400 & - & - & - & - \\
\hline Hg0 vs. Hg0.05 & 1.00 & 0.100 & -0.01 & 0.514 & - & - & - & - \\
\hline Hg0.01 vs. Hg0.1 & 1.00 & 0.100 & 0.16 & 0.057 & - & - & - & - \\
\hline Hg0.05 vs. Hg0.1 & 1.00 & 0.100 & 0.42 & 0.029 & - & - & - & - \\
\hline $\mathrm{Cd}^{2+}$ (global test) & - & - & - & - & 0.76 & 0.001 & 0.79 & 0.001 \\
\hline CdO vs. CdO.1 & - & - & - & - & 0.85 & 0.100 & 0.26 & 0.076 \\
\hline CdO vs. Cd0.5 & - & - & - & - & 0.52 & 0.100 & 0.25 & 0.114 \\
\hline CdO vs. Cd1 & - & - & - & - & 1.00 & 0.100 & 0.85 & 0.005 \\
\hline Cd0.1 vs. Cd0.5 & - & - & - & - & 0.63 & 0.100 & 0.02 & 0.429 \\
\hline Cd0.1 vs. Cd1 & - & - & - & - & 1.00 & 0.100 & 0.46 & 0.057 \\
\hline Cd0.5 vs. Cd1 & - & - & - & - & 1.00 & 0.100 & 0.77 & 0.029 \\
\hline
\end{tabular}

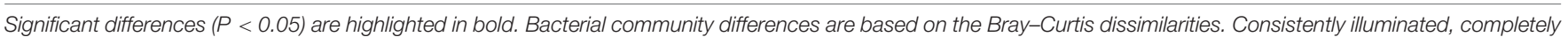
dark, and $12 \mathrm{h:12} \mathrm{h}$ alternation of light and dark conditions referred to as LL, DD and LD; oxytetracycline concentrations of 0, 1,10 , and 20 mg/L referred to as OTC0,

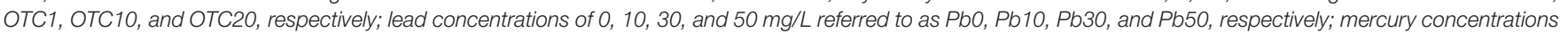

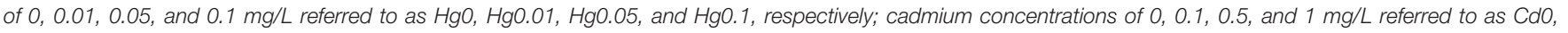
Cd0.1, Cd0.5, and Cd1, respectively.

Verrucomicrobia (11.3\%), and Firmicutes (0.53\%) were relatively minor. The proteobacterial DRB belonged to proteobacterial classes (Gammaproteobacteria, Alphaproteobacteria, and Betaproteobacteria), of which Gammaproteobacteria was the most abundant in P. bursaria and E. vannus, accounting for 37.1 and $71.9 \%$, respectively. The DRB of Alphaproteobacteria showed similar proportions in these two ciliates (10.3 and $13.4 \%)$, whereas the relative abundance of Betaproteobacteria was much higher in the P. bursaria (13.8\%) than in E. vannus (2.1\%) (Figure 2).

A total of 84 OTUs were obtained for the DRB of these two ciliate species, of which 72 OTUs showed high sequence identity (97-100\%) with described species, and the remaining 15 OTUs had sequence similarities lower than 97\% (Table 2). The Proteobacteria OTUs were prevalent in the DRB assemblages of both ciliates, accounting for $81 \%$ of the OTUs observed (Figure 2 


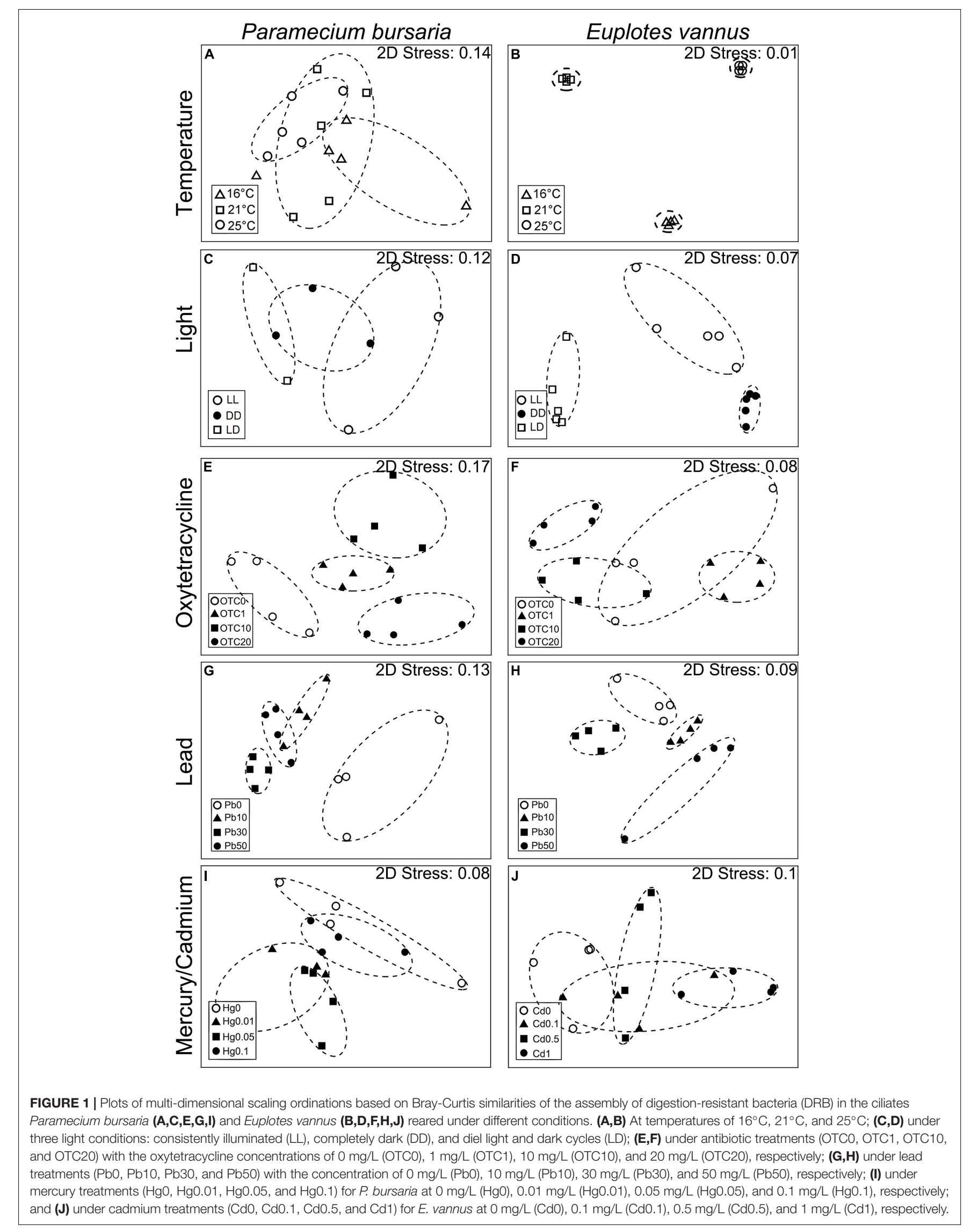


A Parameciun bursaria

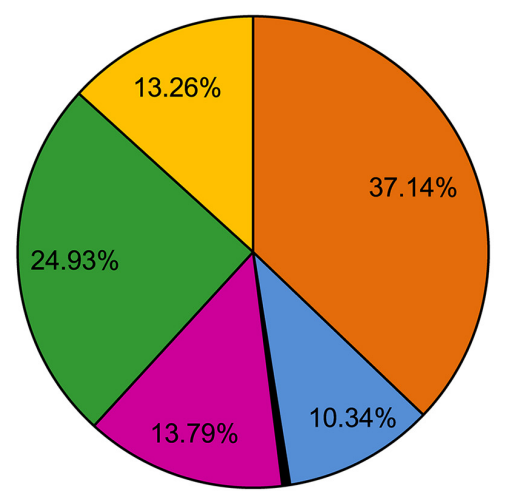

B

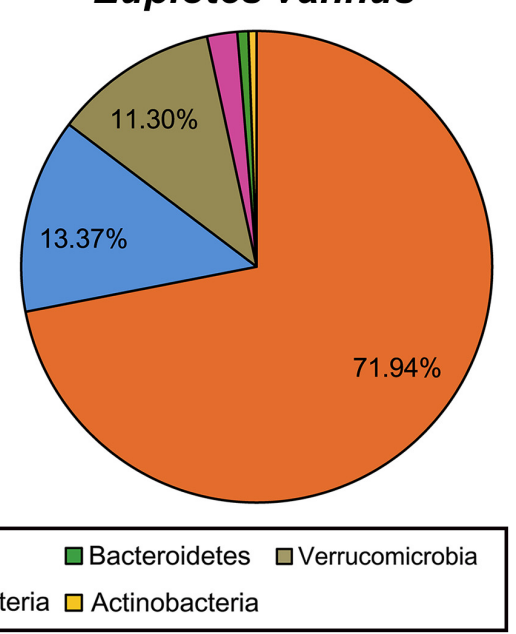

FIGURE 2 | A pie chart showing the phylogenetic composition of digestion-resistant bacteria (DRB) in association with the ciliated protozoa Paramecium bursaria (A) and Euplotes vannus (B).

and Table 2). Gammaproteobacteria (41) also dominated in terms of OTU number, which was about three times the amount of Alphaproteobacteria (14) and Betaproteobacteria (13).

A majority of Gammaproteobacteria OTUs were mostly affiliated with the genera Pseudoalteromonas (13 OTUs), Aestuaribacter (5 OTUs), Alteromonas (5 OTUs), and Pseudomonas (5 OTUs). The phylotypes of the former three genera were exclusively detected in the marine ciliate E. vannus, whereas the phylotypes of Pseudomonas were detected in the DRB assemblages of both P. bursaria and E. vannus (Table 2). Nevskia (2 OTUs) and Spongiibacter (2 OTUs) had fewer OTUs, and Aeromonas, Acinetobacter, Alcanivorax, Escherichia, Haemophilus, Psychrosphaera, Serratia, and Vibrio were each represented with a single OTU (Table 2).

The most frequently occurring OTUs from DRB in the Alphaproteobacteria were affiliated with the family Rhodobacteraceae (5 OTUs, with five genera Thalassococcus, Nautella, Ruegeria, Paracoccus, and Sulfitobacter) (Table 2). The phylotypes of the family Caulobacteraceae were represented by 4 OTUs and two genera, Caulobacter and Asticcacaulis, which were abundant in the DRB assemblages of $P$. bursaria under the light treatments (Table 2). Two OTUs belonged to the genus Sphingomonas and occurred in both ciliates. The remaining three alphaproteobacterial OTUs were Methylobacterium, Phyllobacterium, and Agrobacterium, which were all associated with the P. bursaria. Nearly all of the Betaproteobacteria OTUs were recovered from $P$. bursaria, except for one OTU (Pelomonas sp.) detected in both two ciliate species (Table 2). Other OTUs were affiliated with the genera Aquabacterium, Curvibacter, Hydrogenophaga, Janthinobacterium, Methylophilus, Polynucleobacter, Limnobacter, Ralstonia, and Variovorax (Table 2).

There were 10 OTUs of DRB affiliated with the phylum Bacteroidetes. These included two OTUs of Arcicella and another two of Flectobacillus, both of which are members of the family
Cytophagaceae (Table 2). Three OTUs of Flavobacteriaceae were closely related to Ochrovirga and Flavobacterium. Two OTUs were members of Cryomorphaceae: one was affiliated with Fluviicola, and the other could represent a new genus within this family (Figure 3A), exhibiting a 90\% similarity with the $16 \mathrm{~S}$ rRNA gene of Vicingus serpentipes (Table 2). The OTU sharing an $88 \%$ sequence similarity with Crocinitomix catalasitica was confirmed to be a member of the order Flavobacteriales but remained unresolved at the family level in the ML and Bayesian trees (Figure 3A). The remaining OTU of this phylum was closely related to Algoriphagus (Table 2).

The actinobacterial DRB were represented by two OTUs affiliated with Micrococcus and Propionibacterium (Table 2). Another two OTUs were related to Anaerocolumna and Peptoniphilus (phylum Firmicutes), which were only detected in the freshwater ciliate (Table 2). A single verrucomicrobial OTU, which was associated with the marine ciliate E. vannus, shared a low sequence similarity (89\%) with Ruficoccus amylovorans (family Puniceicoccaceae), likely representing a new genus (Figure 3B and Table 2).

\section{Warming Effect on the Assemblage Structure of DRB}

Analysis of the clone libraries indicated that the DRB assemblage in P. bursaria was dominated by Pseudomonas spp., of which the relative abundance gradually increased from 50\% to $65 \%$ and $76 \%$ with increasing temperature (Table 2 and Figure 4A). Flavobacterium, Peptoniphilus, and Micrococcus, though not abundant, were only detected in the warmest treatment for the freshwater ciliate. In contrast, the DRB assemblage in E. vannus was generally dominated by Pseudoalteromonas spp., of which the relative abundance decreased from $74 \%$ to $40 \%$ with temperature. The relative abundance of Vibrio sp. was minor (2.7\%) during the treatment at $16^{\circ} \mathrm{C}$ but became abundant $(50 \%)$ at $25^{\circ} \mathrm{C}$ (Table 2 
TABLE 2 | Detection and classification of digestion-resistant bacteria (DRB) in Paramecium bursaria (P) and Euplotes vannus (E) in various temperature, light, antibiotic, and heavy metal treatments.

\begin{tabular}{|c|c|c|c|c|c|c|c|}
\hline OTU ID & Accession number & $\begin{array}{l}\text { Closest matched species (accession } \\
\text { number) }\end{array}$ & Coverage (\%) & Identity (\%) & Classification & Treatment & Ciliate \\
\hline OTU1 & MH556452 & Aestuariibacter sp. strain 12C24 (KU963301) & 100 & $99-100$ & Gamma, Alteromonadaceae & $\begin{array}{l}25^{\circ} \mathrm{C} ; \text { LD; OTC0, OTC1, } \\
\text { OTC10; Pb10, Pb30, Pb50; } \\
\text { Cd0, Cd0.1, Cd0.5, Cd1.0 }\end{array}$ & E \\
\hline OTU2 & MH556787 & Aestuariibacter halophilus (LC221844) & 100 & 99 & Gamma, Alteromonadaceae & LD; OTC1, OTC20 & E \\
\hline OTU3 & MH556783 & Aestuariibacter sp. Bh22 (LN897328) & 100 & 94 & Gamma, Alteromonadaceae & Отс20 & E \\
\hline OTU4 & MH556703 & $\begin{array}{l}\text { Aestuariibacter aggregatus strain WH169 } \\
\text { (MH414455) }\end{array}$ & 100 & 97 & Gamma, Alteromonadaceae & OTC20 & E \\
\hline OTU5 & MH556467 & Aestuariibacter sp. strain 12C24 (KU963301) & 100 & $97-98$ & Gamma, Alteromonadaceae & OTC0; CdO.1 & $\mathrm{E}$ \\
\hline OTU6 & MH556726 & Alteromonas sp. H86 (FJ903192) & 100 & 98 & Gamma, Alteromonadaceae & OTC1 & E \\
\hline OTU7 & MH556162 & Alteromonas sp. M71_D24 (FM992713) & 100 & 95 & Gamma, Alteromonadaceae & LD & E \\
\hline OTU8 & MH556150 & Alteromonas sp. SPB-8 (DQ412077) & 93 & 98 & Gamma, Alteromonadaceae & LD & E \\
\hline OTU9 & MH556135 & Alteromonas sp. AKA07-1 (AB571941) & 100 & $97-98$ & Gamma, Alteromonadaceae & LD; OTCO, OTC1 & E \\
\hline OTU10 & MH556153 & $\begin{array}{l}\text { Alteromonas tagae strain BCRC } 17571 \\
\text { (NR_043977) }\end{array}$ & 100 & $97-99$ & Gamma, Alteromonadaceae & LD, LL & E \\
\hline OTU11 & MH556079 & $\begin{array}{l}\text { Pseudoalteromonas phenolica strain JCM } \\
21460 \text { (NR_113299) }\end{array}$ & 100 & $96-97$ & Gamma, Pseudoalteromonadaceae & LL, DD & $E$ \\
\hline OTU12 & MH556080 & Pseudoalteromonas sp. 03/034 (AJ874351) & 100 & $96-98$ & Gamma, Pseudoalteromonadaceae & LL & E \\
\hline OTU13 & MH556095 & $\begin{array}{l}\text { Psychrosphaera saromensis strain DL8-2 } \\
\text { (KF146527) }\end{array}$ & 100 & $97-98$ & Gamma, Pseudoalteromonadaceae & LL, DD, LD & E \\
\hline OTU14 & MH556118 & Pseudoalteromonas sp. 03/034 (AJ874351) & 100 & 99 & Gamma, Pseudoalteromonadaceae & $16^{\circ} \mathrm{C} ; \mathrm{DD}, \mathrm{LL}$ & E \\
\hline OTU15 & MH556233 & $\begin{array}{l}\text { Pseudoalteromonas piscicida strain MCCB } 201 \\
\text { (KF880965) }\end{array}$ & 100 & 98-99 & Gamma, Pseudoalteromonadaceae & $25^{\circ} \mathrm{C}$ & E \\
\hline OTU16 & MH556167 & Pseudoalteromonas sp. 01/121 (AJ874345) & 100 & $98-100$ & Gamma, Pseudoalteromonadaceae & $16^{\circ} \mathrm{C}, 21^{\circ} \mathrm{C} ; \mathrm{DD}, \mathrm{LL}$ & E \\
\hline OTU17 & MH556181 & Pseudoalteromonas sp. A28 (AF227238) & 100 & 95 & Gamma, Pseudoalteromonadaceae & $16^{\circ} \mathrm{C}$ & E \\
\hline OTU18 & MH556191 & Pseudoalteromonas sp. BSw21650 (JF697294) & 100 & 98-99 & Gamma, Pseudoalteromonadaceae & $16^{\circ} \mathrm{C}, 21^{\circ} \mathrm{C}$ & $E$ \\
\hline OTU19 & MH556216 & Pseudoalteromonas sp. CF6-1 (FJ169996) & 100 & 96 & Gamma, Pseudoalteromonadaceae & $21^{\circ} \mathrm{C}$ & E \\
\hline OTU20 & MH556254 & Pseudoalteromonas sp. HK41 (HQ343275) & 100 & $97-98$ & Gamma, Pseudoalteromonadaceae & $25^{\circ} \mathrm{C}$ & E \\
\hline OTU21 & MH556169 & Pseudoalteromonas sp. KASP34 (KU647930) & 99 & 99 & Gamma, Pseudoalteromonadaceae & $16^{\circ} \mathrm{C}$ & E \\
\hline OTU22 & MH556210 & Pseudoalteromonas sp. S1649 (FJ457154) & 100 & $98-99$ & Gamma, Pseudoalteromonadaceae & $16^{\circ} \mathrm{C}, 21^{\circ} \mathrm{C} ; \mathrm{LL}, \mathrm{DD}$ & E \\
\hline OTU23 & MH556188 & Pseudoalteromonas sp. S511-1 (AB029824) & 100 & $97-98$ & Gamma, Pseudoalteromonadaceae & $16^{\circ} \mathrm{C}, 21^{\circ} \mathrm{C}$ & E \\
\hline OTU24 & MH556236 & Pseudoalteromonas viridis (AB681561) & 100 & 95 & Gamma, Pseudoalteromonadaceae & $25^{\circ} \mathrm{C}$ & E \\
\hline OTU25 & MH556186 & $\begin{array}{l}\text { Algicola bacteriolytica strain MR32e } \\
\text { (HQ439519) }\end{array}$ & 99 & 95 & Gamma, Pseudoalteromonadaceae & $16^{\circ} \mathrm{C}$ & $E$ \\
\hline OTU26 & MH555990 & $\begin{array}{l}\text { Pseudomonas synxantha strain NBRC } 3913 \\
\text { (NR_113583) }\end{array}$ & 100 & $98-99$ & Gamma, Pseudomonadaceae & $16^{\circ} \mathrm{C} ; \mathrm{OTC} 1$ & $E, P$ \\
\hline OTU27 & MH555987 & $\begin{array}{l}\text { Pseudomonas psychrophila strain P270 } \\
\text { (KC904093) }\end{array}$ & 100 & $99-100$ & Gamma, Pseudomonadaceae & $\begin{array}{l}16^{\circ} \mathrm{C}, 21^{\circ} \mathrm{C}, 25^{\circ} \mathrm{C} ; \mathrm{OTC} 1 \\
\text { OTC10, OTC20 }\end{array}$ & $P$ \\
\hline
\end{tabular}


TABLE 2 | Continued

\begin{tabular}{|c|c|c|c|c|c|c|c|}
\hline OTU ID & Accession number & $\begin{array}{l}\text { Closest matched species (accession } \\
\text { number) }\end{array}$ & Coverage (\%) & Identity (\%) & Classification & Treatment & Ciliate \\
\hline OTU28 & MH556036 & Pseudomonas sp. K3R3.1A (KC433646) & 100 & 99 & Gamma, Pseudomonadaceae & $\begin{array}{l}21^{\circ} \mathrm{C}, 25^{\circ} \mathrm{C} ; \mathrm{HgO} .01 \text {; ОTCO, } \\
\text { OTC10, OTC20 }\end{array}$ & $P$ \\
\hline OTU29 & MH556031 & $\begin{array}{l}\text { Pseudomonas fluorescens strain ex17 } \\
\text { (KF317887) }\end{array}$ & 100 & $99-100$ & Gamma, Pseudomonadaceae & $\begin{array}{l}16^{\circ} \mathrm{C}, 21^{\circ} \mathrm{C}, 25^{\circ} \mathrm{C} \text {; OTCO, } \\
\mathrm{OTC} 1, \mathrm{OTC} 10 ; \mathrm{Pb} 10 ; \mathrm{HgO} \\
\mathrm{HgO} 01 \text {, } \mathrm{HgO} .1\end{array}$ & $E, P$ \\
\hline OTU30 & MH556663 & Pseudomonas sp. R02 (KT890300) & 100 & 98 & Gamma, Pseudomonadaceae & OTC20 & $P$ \\
\hline OTU31 & MH555949 & Nevskia ramosa strain Soe1 (NR_025269) & 99 & $98-99$ & Gamma, Sinobacteraceae & $\mathrm{DD}, \mathrm{LD}, \mathrm{LL}$ & $P$ \\
\hline OTU32 & MH555974 & Nevskia ramosa (AJ001343) & 99 & 95 & Gamma, Sinobacteraceae & DD & $P$ \\
\hline OTU33 & MH556800 & Spongiibacter marinus (AB985586) & 100 & $96-97$ & Gamma, Spongiibacteraceae & отс10 & E \\
\hline OTU34 & MH556747 & Spongiiibacter marinus (AB985580) & 100 & 99 & Gamma, Spongiibacteraceae & OTC10, ОTC20; LD & E \\
\hline OTU35 & MH556281 & Escherichia coli strain Y38 (JN578647) & 100 & 99 & Gamma, Enterobacteriaceae & $\mathrm{HgO}, \mathrm{HgO} .05, \mathrm{HgO} .1$ & $P$ \\
\hline OTU36 & MH556409 & $\begin{array}{l}\text { Serratia proteamaculans strain PW172 } \\
\text { (JF494823) }\end{array}$ & 100 & 99 & Gamma, Enterobacteriaceae & $\mathrm{Pb} 50$ & $P$ \\
\hline OTU37 & MH556249 & Vibrio neocaledonicus strain MS1 (KJ841877) & 100 & 99 & Gamma, Vibrionaceae & $16^{\circ} \mathrm{C}, 25^{\circ} \mathrm{C}$ & E \\
\hline OTU38 & MH556000 & Aeromonas caviae strain AH08 (KU975030) & 100 & 99 & Gamma, Aeromonadaceae & $16^{\circ} \mathrm{C}$ & $P$ \\
\hline OTU39 & MH556788 & Alcanivorax sp. MCCC 1A00973 (KU681505) & 100 & 99 & Gamma, Alcanivoracaceae & Отс20 & E \\
\hline OTU40 & MH556230 & Acinetobacter junii strain B2w (KX058411) & 100 & 99 & Gamma, Moraxellaceae & $\begin{array}{l}21^{\circ} \mathrm{C} ; \mathrm{LD} ; \mathrm{HgO} .01, \mathrm{HgO} .05 \\
\text { OTCO, OTC10 }\end{array}$ & $E, P$ \\
\hline OTU41 & MH556303 & Haemophilus sp. oral (AY005034) & 100 & 99 & Gamma, Pasteurellaceae & $\mathrm{HgO} 0.01$ & $P$ \\
\hline OTU42 & MH556285 & $\begin{array}{l}\text { Asticcacaulis excentricus strain CB } 48 \\
\text { (NR_114730) }\end{array}$ & $98-100$ & 99 & Alpha, Caulobacteraceae & $\mathrm{HgO}, \mathrm{Hg} 0.1$ & $P$ \\
\hline OTU43 & MH555955 & Asticcacaulis sp. clone Sa4_4.4 (GQ181156) & 100 & 98 & Alpha, Caulobacteraceae & LD, DD, LL & $P$ \\
\hline OTU44 & MH555978 & Caulobacter sp. BBCT11 (DQ337547) & 100 & 96 & Alpha, Caulobacteraceae & DD & $P$ \\
\hline OTU45 & MH555956 & Caulobacter sp. DNA (AJ227760) & 100 & 99 & Alpha, Caulobacteraceae & $\mathrm{LL}$ & $P$ \\
\hline OTU46 & MH556716 & Thalassococcus sp. KU27F5 (AB636146) & 100 & 99 & Alpha, Rhodobacteraceae & OTC1 & E \\
\hline OTU47 & MH556768 & Nautella sp. A04V (LC094988) & 100 & $97-99$ & Alpha, Rhodobacteraceae & LL; OTC0, OTC1, OTC10 & E \\
\hline OTU48 & MH556144 & $\begin{array}{l}\text { Ruegeria pelagia strain NBRC } 102038 \\
\text { (NR_114024) }\end{array}$ & 100 & $99-100$ & Alpha, Rhodobacteraceae & LL, LD & E \\
\hline OTU49 & MH556262 & Paracoccus sp. 17 (KR108387) & 100 & 99 & Alpha, Rhodobacteraceae & $\mathrm{HgO}$ & $P$ \\
\hline OTU50 & MH556666 & Sulfitobacter sp. QD214-NF102 (KC689801) & 100 & 99 & Alpha, Rhodobacteraceae & ОTС20 & $\mathrm{P}$ \\
\hline OTU51 & MH555995 & $\begin{array}{l}\text { Sphingomonas abaci strain SS1-08 } \\
\text { (KU341393) }\end{array}$ & 100 & 99 & Alpha, Sphingomonadaceae & $\begin{array}{l}\text { LD; } 16^{\circ} \mathrm{C} \text {; OTCO, OTC1, } \\
\text { OTC10, OTC20 }\end{array}$ & $E, P$ \\
\hline OTU52 & MH556759 & $\begin{array}{l}\text { Sphingomonas roseiflava strain MK341 } \\
\text { (NR_117716) }\end{array}$ & 100 & 97 & Alpha, Sphingomonadaceae & OTC10 & E \\
\hline OTU53 & MH556325 & Methylobacterium sp. 20 (JF905619) & 100 & 99 & Alpha, Methylobacteriaceae & $\mathrm{HgO} .05$ & $P$ \\
\hline OTU54 & MH556314 & $\begin{array}{l}\text { Phyllobacterium myrsinacearum isolate } \\
\text { OTU-a22 (KJ147062) }\end{array}$ & 100 & 99 & Alpha, Phyllobacteriaceae & $16^{\circ} \mathrm{C} ; \mathrm{HgO} .05$ & $P$ \\
\hline
\end{tabular}


TABLE 2 | Continued

\begin{tabular}{|c|c|c|c|c|c|c|c|}
\hline OTU ID & Accession number & $\begin{array}{l}\text { Closest matched species (accession } \\
\text { number) }\end{array}$ & Coverage $(\%)$ & Identity (\%) & Classification & Treatment & Ciliate \\
\hline OTU55 & MH556037 & $\begin{array}{l}\text { Agrobacterium rhizogenes strain IV } \\
\text { (HM582866) }\end{array}$ & 100 & 99 & Alpha, Rhizobiaceae & $21^{\circ} \mathrm{C}$ & $\mathrm{P}$ \\
\hline OTU56 & MH556569 & Pelomonas sp. clone 35Fe00 (KF287732) & 100 & 99 & Beta, Comamonadaceae & $\begin{array}{l}\mathrm{Pb} 30, \mathrm{~Pb} 50 ; \mathrm{Hg} 0.01 ; \mathrm{Cd} 0.1 \text {, } \\
\mathrm{Cd} 1.0\end{array}$ & $E, P$ \\
\hline OTU57 & MH556333 & Hydrogenophaga sp. 7B-224 (KF441666) & 99 & 98 & Beta, Comamonadaceae & $\mathrm{Hg} 0.1$ & $\mathrm{P}$ \\
\hline OTU58 & MH555972 & $\begin{array}{l}\text { Curvibacter lanceolatus strain NBRC } 103051 \\
\text { (NR_114201) }\end{array}$ & 100 & $98-99$ & Beta, Comamonadaceae & LL, LD, DD & $P$ \\
\hline OTU59 & MH555959 & Variovorax paradoxus (HQ845986) & 100 & 99 & Beta, Comamonadaceae & LL, LD & $\mathrm{P}$ \\
\hline OTU60 & MH556621 & Ralstonia sp. S1SM82 (KT183537) & 100 & 99 & Beta, Burkholderiaceae & Отсо & $P$ \\
\hline OTU61 & MH556271 & Polynucleobacter acidiphobus (AB599874) & 100 & 99 & Beta, Burkholderiaceae & $\mathrm{HgO}$ & $P$ \\
\hline OTU62 & MH556312 & Limnobacter sp. KNF002 (AB426551) & 100 & 99 & Beta, Burkholderiaceae & $\mathrm{Hg} 0.05$ & $P$ \\
\hline OTU63 & MH556310 & $\begin{array}{l}\text { Aquabacterium commune strain B8 } \\
\text { (NR_024875) }\end{array}$ & 100 & $98-99$ & Beta, Burkholderiales & $\mathrm{Hg} 0.01, \mathrm{HgO} 0.05$ & $P$ \\
\hline OTU64 & MH556265 & $\begin{array}{l}\text { Aquabacterium fontiphilum strain K10 } \\
\text { (KT345664) }\end{array}$ & 100 & 99 & Beta, Burkholderiales & $\mathrm{HgO}$ & $P$ \\
\hline OTU65 & MH556313 & $\begin{array}{l}\text { Aquabacterium fontiphilum strain CS-6 } \\
\text { (NR_044322) }\end{array}$ & 98 & 98 & Beta, Burkholderiales & $\mathrm{HgO} .05$ & $P$ \\
\hline OTU66 & MH556272 & Limnobacter sp. strain DRY11W (MH463959) & 100 & 94 & Beta, Burkholderiales & $\mathrm{HgO}$ & $P$ \\
\hline OTU67 & MH556266 & $\begin{array}{l}\text { Methylophilus methylotrophus strain NCIMB } \\
10515 \text { (NR_041257) }\end{array}$ & 99 & 99 & Beta, Methylophilaceae & $\mathrm{HgO}$ & $P$ \\
\hline OTU68 & MH556063 & $\begin{array}{l}\text { Janthinobacterium sp. TP-Snow-C76 } \\
\text { (KC987006) }\end{array}$ & 100 & 99 & Beta, Oxalobacteraceae & $16^{\circ} \mathrm{C}, 25^{\circ} \mathrm{C}$ & $P$ \\
\hline OTU69 & MH556619 & Arcicella aquatica strain NO-502 (NR_029000) & 100 & 99 & Bacteroidetes, Cytophagaceae & Отсо & $P$ \\
\hline OTU70 & MH556411 & Arcicella rigui strain HMF3820 (KT983986) & 100 & 96 & Bacteroidetes, Cytophagaceae & $\mathrm{Pb} 50$ & $P$ \\
\hline OTU71 & MH556371 & Flectobacillus sp. WG3 (FN547417) & 100 & $98-99$ & Bacteroidetes, Cytophagaceae & $\begin{array}{l}\mathrm{Pb} 10, \mathrm{~Pb} 30, \mathrm{~Pb} 50 ; \mathrm{HgO} \\
\mathrm{Hg0} 0.05\end{array}$ & $P$ \\
\hline OTU72 & MH556345 & $\begin{array}{l}\text { Flectobacillus rhizosphaerae strain JC289 } \\
\text { (NR_137382) }\end{array}$ & 96 & 95 & Bacteroidetes, Cytophagaceae & $\mathrm{Pb} 10$ & $P$ \\
\hline OTU73 & MH556228 & Ochrovirga pacifica (JN596241) & 97 & 95 & Bacteroidetes, Flavobacteriaceae & $21^{\circ} \mathrm{C}$ & E \\
\hline OTU74 & MH556330 & $\begin{array}{l}\text { Flavobacterium fontis strain MIC3010 } \\
\text { (NR_109522) }\end{array}$ & 96 & 99 & Bacteroidetes, Flavobacteriaceae & $\mathrm{Hg} 0.1$ & $P$ \\
\hline OTU75 & MH556040 & $\begin{array}{l}\text { Flavobacterium sp. T3L.05.LWF.W.Kidney.D } \\
\text { (JX287789) }\end{array}$ & 98 & $99-100$ & Bacteroidetes, Flavobacteriaceae & $25^{\circ} \mathrm{C}$ & $P$ \\
\hline OTU76 & MH556400 & $\begin{array}{l}\text { Fluviicola hefeinensis strain MYL-8 } \\
\text { (NR_133750) }\end{array}$ & 100 & 98 & Bacteroidetes, Cryomorphaceae & $\mathrm{Pb} 50$ & $P$ \\
\hline OTU77 & MH556320 & $\begin{array}{l}\text { Vicingus serpentipes strain ANORD5 } \\
\text { (NR_159281) }\end{array}$ & 100 & 90 & Bacteroidetes, Cryomorphaceae & $\mathrm{Hg} 0.05$ & $\mathrm{P}$ \\
\hline
\end{tabular}




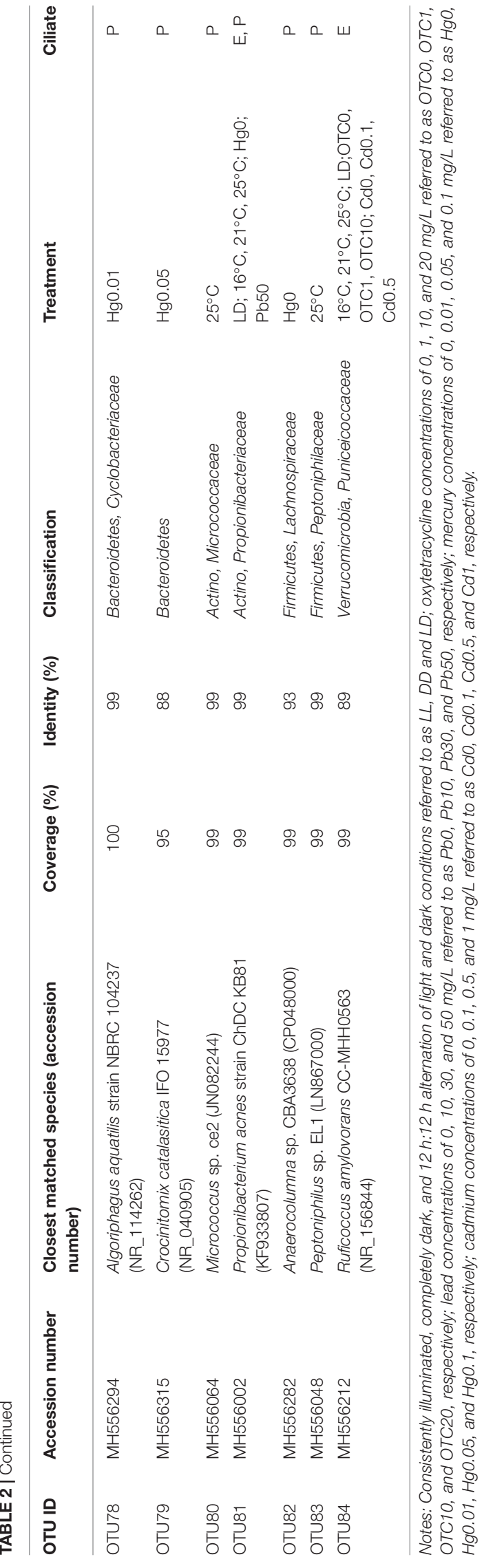

and Figure 4A). Ruficoccus-like species (Verrucomicrobia) were consistently present, accounting for a small portion (3.3$13.5 \%)$ of the DRB in association with E. vannus. Nevertheless, warming induced a gradual decrease in its relative abundance. Aestuariibacter and Pseudomonas spp. were present only in the warmest treatment of the Euplotes (Figure 4A).

\section{Variations in the Bacterial Assemblages Under Photoperiod Conditions}

E. vannus under the condition of LD hosted 9 bacterial genera, which was much more diverse than that in both LL and DD. Structurally, Pseudoalteromonas spp. and Psychrosphaera saromensis dominated under conditions of both LL and DD, whereas Aestuaribacter spp. were the most abundant (31\%) in the LD treatment. Phylotypes of Aestuariibacter, Propionibacterium, Sphingomonas, Spongiibacter, and Ruficoccuslike were detected only under the LD treatment (Figure 4B). In contrast, P. bursaria consistently hosted Nevskia sp. (38-44\%), Curvibacter (25-29\%), and Asticcacaulis (6-25\%). Interestingly, Caulobacter and Variovorax were present but not in LD and DD, respectively (Figure $4 B$ ).

\section{Bacteria Co-resistant to Antibiotic and Digestion}

Sphingomonas and Arcicella species occurred in the DRB of P. bursaria, accounting for $73 \%$ in the control (OTC0). However, these two taxa were not detectable in the OTC treatments. Pseudomonas in the control P. bursaria had a low abundance (19\%) but a high abundance (50\%-100\%) after OTC applications. Also, Sulfitobacter was absent in the control and at a lower dose of OTC but represented $47 \%$ of the DRB in the OTC20 treatment (Figure 5A).

The bacteria associated with $E$. vannus treated with OTC included Aestuaribacter, Acinetobacter, Alcanivorax, Alteromonas, Pseudomonas, Nautella, Thalassococcus, Sphingomonas, Spongiibacter, and Ruficoccus-like Verrucomicrobia (Figure 5A). In contrast to the non-detection of Sphingomonas in P. bursaria at high doses of OTC, this genus occurred with high relative abundance in $E$. vannus treated with 10 and $20 \mathrm{mg} / \mathrm{L}$ of OTC. Both the Aestuariibacter and Ruficoccuslike phylotypes were abundant at the low-dose treatment but were reduced or disappeared at the higher doses (Figure 5A).

\section{DRB Assemblages Changed With the Concentrations of Heavy Metals}

The DRB in the control P. bursaria $(\mathrm{Pb} 0)$ were represented by nine bacterial genera, of which only four were detected in the Pb-treated treatments, including Flectobacillus, Pseudomonas, Fluviicola, and Serratia. Flectobacillus was the most dominant, accounting for $83 \%, 100 \%$, and $64 \%$ in $\mathrm{Pb} 10, \mathrm{~Pb} 30$, and $\mathrm{Pb} 50$, respectively. Fluviicola and Serratia together represented 36\% sequences in $\mathrm{Pb} 50$ (Table 2 and Figure 5B).

There were many DRB persisting in the Hg treatments. These included Acinetobacter, Aquabacterium, Escherichia, Flavobacterium, Hydrogenophaga, Pelomonas, Phyllobacterium, and Pseudomonas. The most abundant at the high dose 
A

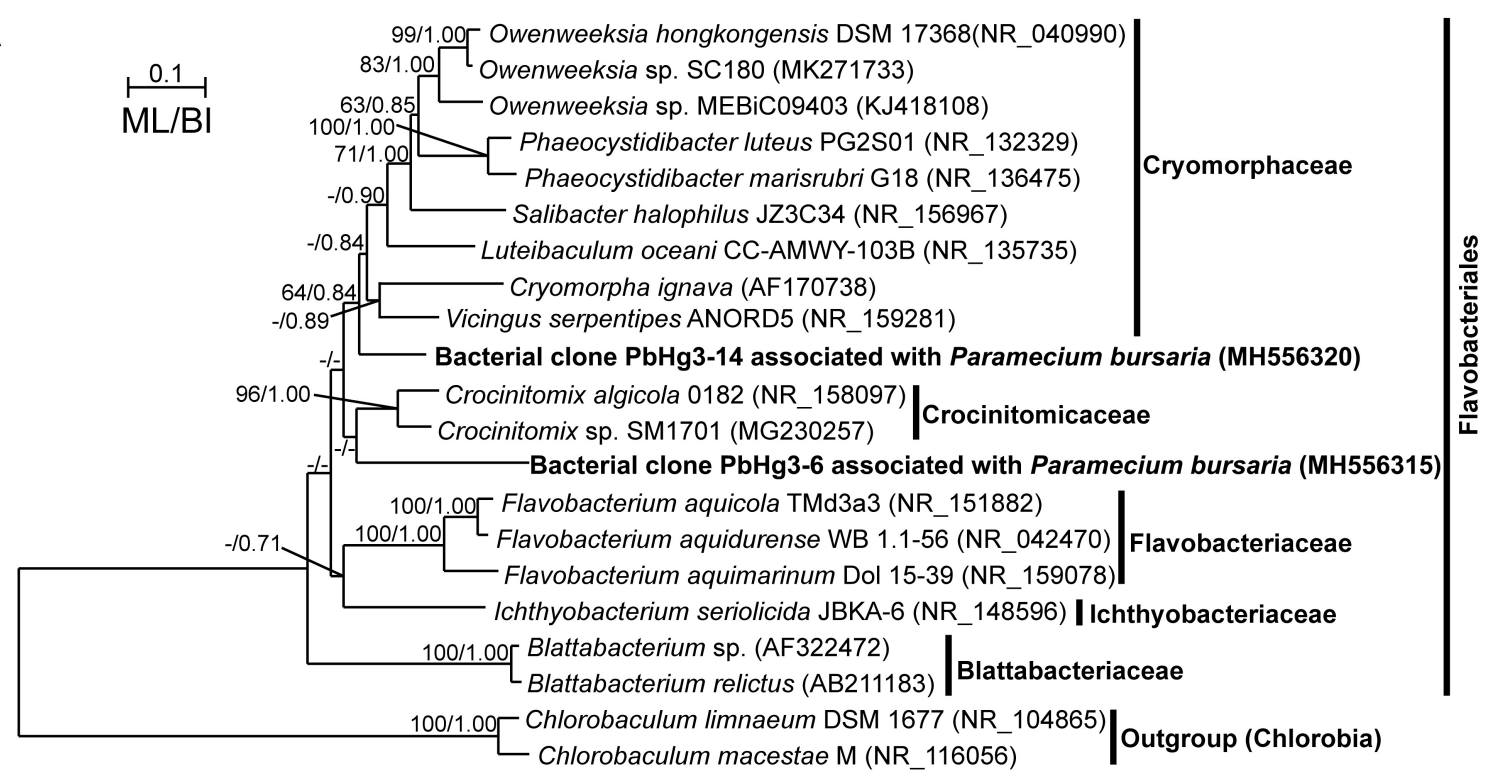

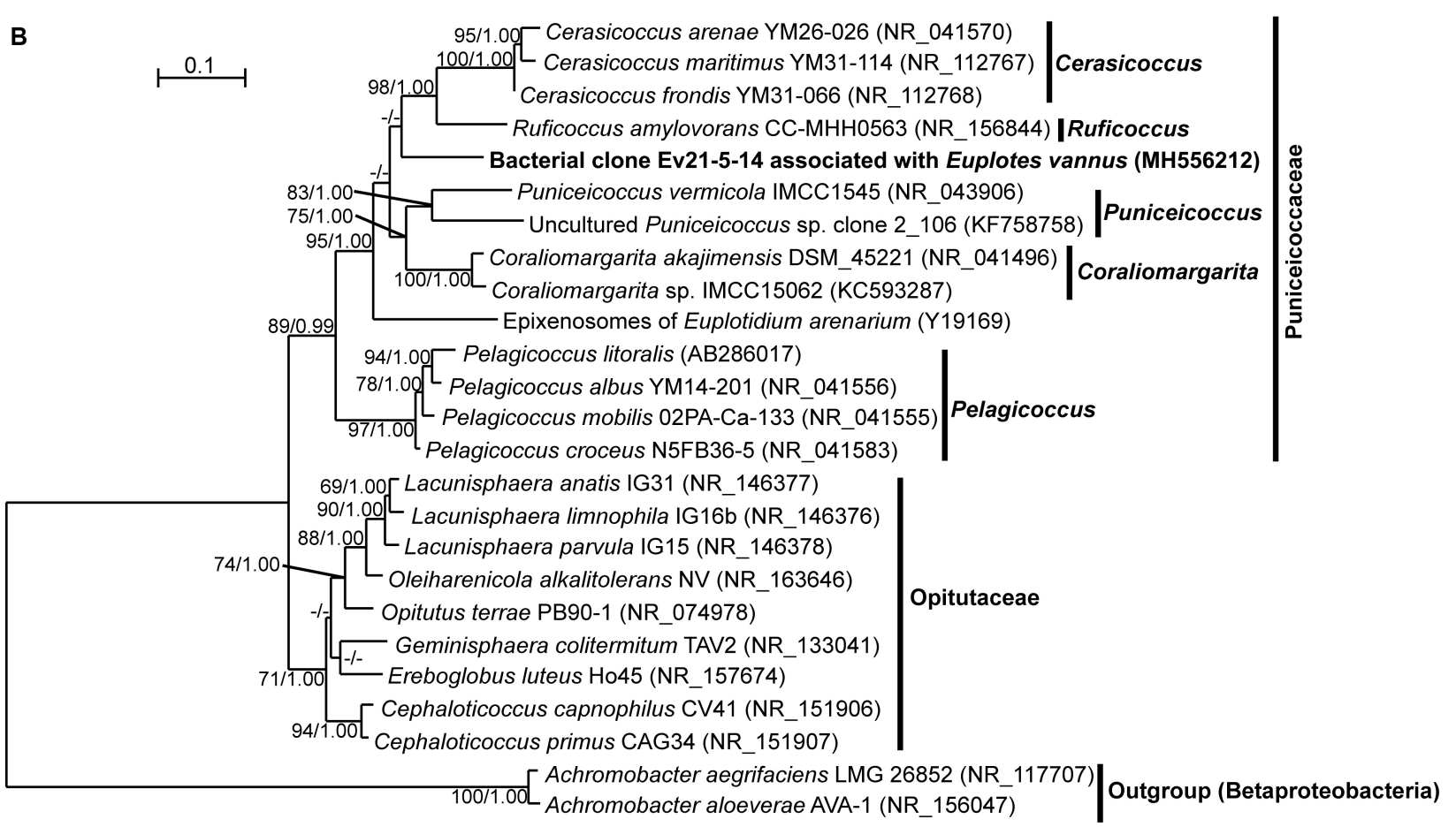

FIGURE 3 | Maximum likelihood (ML) trees based on 16S rRNA genes showing the phylogenetic positions of digestion-resistant bacterial (DRB) phylotypes affiliated with the order Flavobacteriales (A) and with the family Puniceicoccaceae (B). The trees generated using Bayesian Inference (BI) showed similar topologies with the $\mathrm{ML}$ trees. The newly obtained sequences are highlighted in bold. The numbers at the nodes show the bootstrap values and posterior probabilities; only the bootstrap values not lower than $50 \%$ and the posterior probabilities not lower than 0.8 are shown. Accession numbers are provided for all sequences.

of $\mathrm{HgCl}_{2}$ were Hydrogenophaga (42\%) and Escherichia (25\%), whereas Acinetobacter (54\%) and Pseudomonas (13\%) dominated the assemblage in the low dose treatment (Table 2 and Figure 5C).

The DRB assemblage in E. vannus was consistently dominated by a single genus, Aestuariibacter, in both $\mathrm{Pb}$ and Cd treatments (Table 2 and Figures 5B,C). Nevertheless,
Pelomonas (11\%) occurred in $\mathrm{Pb} 30$ and increased to $17 \%$ in $\mathrm{Pb} 50$ (Figure 5B). Furthermore, in the treatments of Cd0.1 and Cd1.0, Pelomonas was also present but with minor proportions (3 and 8\%, respectively). Ruficoccuslike Verrucomicrobia was minor $(3.6 \%)$ in the control but abundant $(\sim 35 \%)$ in both Cd0.1 and Cd0.5 treatments (Table 2 and Figure 5C). 


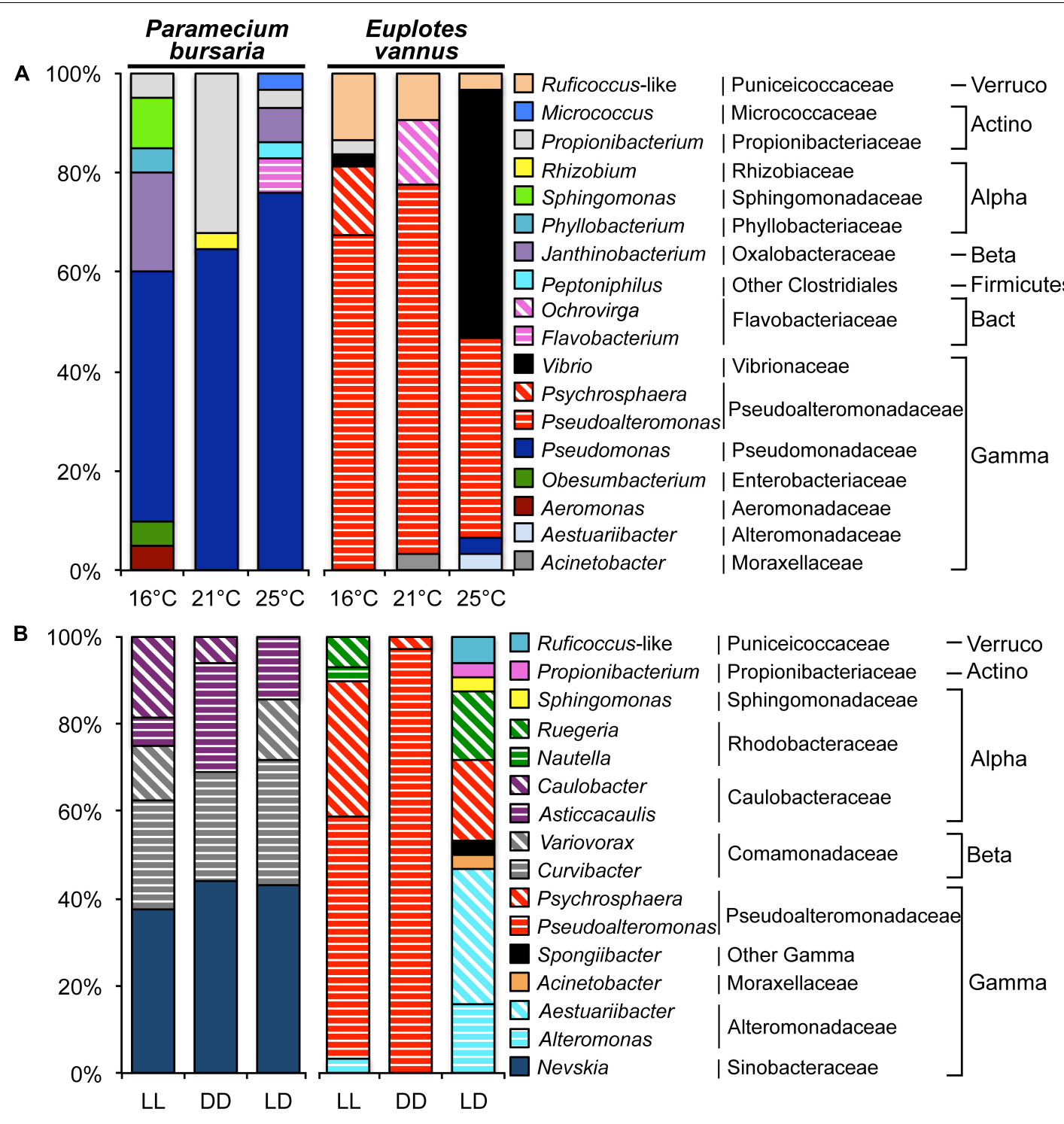

FIGURE 4 | Variations in the assemblage structure of digestion-resistant bacteria (DRB) in association with two ciliates Paramecium bursaria and Euplotes vannus in different treatments. (A) A temperature gradient $\left(16^{\circ} \mathrm{C}, 21^{\circ} \mathrm{C}\right.$, and $\left.25^{\circ} \mathrm{C}\right)$; and (B) light conditions ( $\mathrm{LL}$, DD, and LD represent consistently illuminated, completely dark, and $12 \mathrm{h:12} \mathrm{h}$ alternation of light and dark conditions, respectively). Note that Pseudomonas and Pseudoalteromonas dominated the DRB assemblages in $P$. bursaria and $E$. vannus, with increasing and decreasing relative abundance with temperature, respectively. In different light environments, the composition of DRB in P. bursaria was rather similar, whereas Aestuariibacter and Alteromonas in E. vannus became more important under the condition of light-dark diel cycle. Abbreviations: Actinobacteria (Actino), Alphaproteobacteria (Alpha), Bacteroidetes (Bact), Betaproteobacteria (Beta), Gammaproteobacteria (Gamma), and Verrucomicrobia (Verruco).

\section{DISCUSSION}

By using E. vannus and P. bursaria as models, this study is the first to explicitly investigate how the composition and structure of DRB assemblages vary along various physical and chemical gradients. Overall, our molecular profiling of these two protist species indicated that experimentally manipulated warming, irradiance, antibiotic, and heavy metal stresses significantly influenced the structure of DRB assemblages. This provides evidence that the association between DRB and protists is highly dependent on environmental conditions.
It was conceivable that there were consistently larger variations in the assemblage structure of $\mathrm{DRB}$, relative to those in the environmental bacterioplankton across the treatments of E. vannus (Table 1). This is likely related to the fact that the variations in the environmental factors caused the shifts in the bacterioplankton community structure first (Supplementary Table S1), and then selective ingestion and differential digestion of the engulfed bacterial taxa took place next, which further screened out digestible populations, resulting in the altered assemblage structure of the bacteria (i.e., DRB) that resided in the protistan cells (Table 1 and Figures 4, 5). However, 


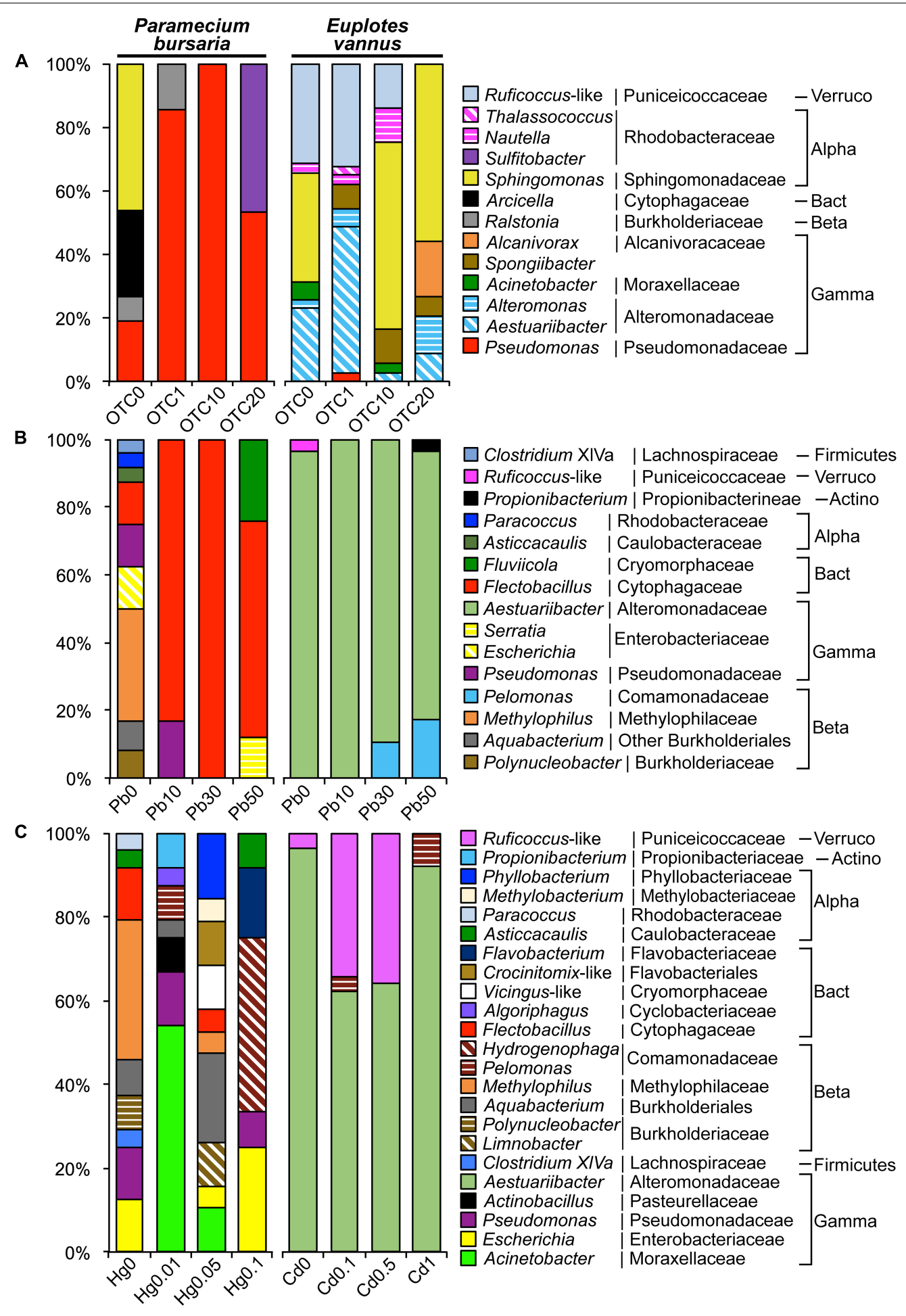

FIGURE $\mathbf{5}$ | Variations in the assemblage structure of digestion-resistant bacteria (DRB) in Paramecium bursaria and Euplotes vannus (A) under antibiotic treatments (OTC0, OTC1, OTC10, and OTC20) with the oxytetracycline concentrations of $0 \mathrm{mg} / \mathrm{L}$ (OTC0), $1 \mathrm{mg} / \mathrm{L}$ (OTC1), $10 \mathrm{mg} / \mathrm{L}$ (OTC10), and $20 \mathrm{mg} / \mathrm{L}$ (OTC20), respectively; (B) under the lead treatments ( $\mathrm{PbO}, \mathrm{Pb} 10, \mathrm{~Pb} 30$, and $\mathrm{Pb50}$ ) with the concentration of $0 \mathrm{mg} / \mathrm{L}$ (Pb0), $10 \mathrm{mg} / \mathrm{L}$ (Pb10), $30 \mathrm{mg} / \mathrm{L}$ (Pb30), and 50 mg/L (Pb50), respectively; (C) under mercury treatments ( $\mathrm{HgO}, \mathrm{HgO} .01, \mathrm{HgO} 05$, and $\mathrm{HgO} .1)$ for P. bursaria at $0 \mathrm{mg} / \mathrm{L}(\mathrm{HgO}), 0.01 \mathrm{mg} / \mathrm{L}$ ( $\mathrm{Hg0} .01), 0.05 \mathrm{mg} / \mathrm{L}(\mathrm{HgO} .05)$, and $0.1 \mathrm{mg} / \mathrm{L}(\mathrm{HgO} .1)$, respectively; (C) and under cadmium treatments (Cd0, Cd0.1, Cd0.5, and Cd1) for E. vannus at $0 \mathrm{mg} / \mathrm{L}(\mathrm{Cd0}), 0.1 \mathrm{mg} / \mathrm{L}$ (Cd0.1), $0.5 \mathrm{mg} / \mathrm{L}$ (Cd0.5), and $1 \mathrm{mg} / \mathrm{L}$ (Cd1), respectively. Note that Pseudomonas, Sulfitobacter, and Sphingomonas were highly antibiotic-resistant. Flectobacillus and Fluviicola were highly Pb-resistant. Hydrogenophaga was Hg-resistant, and both Aestuariibacter and Pelomonas were co-resistant to Pb and Cd. Abbreviations: Actinobacteria (Actino), Alphaproteobacteria (Alpha), Bacteroidetes (Bact), Betaproteobacteria (Beta), Gammaproteobacteria (Gamma), and Verrucomicrobia (Verruco). 
this reasoning cannot explain the results for $P$. bursaria, in which the assemblage structure of DRB across multiple environmental gradients appeared to be less variable than that of environmental bacterioplankton. The contrasting variability of DRB in P. bursaria and E. vannus may be related to their nutritional models (mixotrophy vs. heterotrophy), in which the mixotrophs have a highly flexible physiology to cope with the changing environment by shifting between phototrophic and phagotrophic lifestyles. The characteristic intracellular environment of mixotrophs thus selects a narrow spectrum of bacterial species to reside intracellularly.

The selection of DRB in all the treatments investigated in this study may be related to the direct influence of environmental factors and pollution stresses on the physiology and biochemistry of the protists, which may affect the interactions between the predators and preys. At a higher temperature (not higher than the optimal temperature), a protist usually has a high growth rate (Montagnes and Weisse, 2000), ingestion rate (Izaguirre et al., 2012), and digestion rate (Sherr et al., 1988). Compared to the light condition, protozoan ingestion rate determined by using fluorescently labeled prey was lower under the dark conditions (Izaguirre et al., 2012). Light availability is important for mixotrophs. For example, Chlorella symbionts provide photosynthetic products to their host under light conditions (Shibata et al., 2016), but they lost their ability to resist host digestion in darkness (Kodama and Fujishima, 2014). Heavy metals also affect protistan ingestion rates (Al-Rasheid and Sleigh, 1994) and physiology (Kim et al., 2011).

The bacterial species associated with the protistan grazers may be related to not only the life stage and physiology of ciliates (Xu et al., 2020), but also depend on the biological nature (e.g., digestibility) of these bacteria (Zou et al., 2020), which are more or less reflected by their phylogenetic classification. Our analyses of clone libraries revealed that the overall DRB assemblage composition in E. vannus across all treatments was dominated by Gamma- and Alphaproteobacteria, which was consistent with previous reports for a range of marine ciliate species and picoeukaryotes (Farnelid et al., 2016; Gong et al., 2016). However, this compositional trait of DRB was not applicable for the DRB in the freshwater species P. bursaria, in which Gammaproteobacteria, Bacteroidetes, and Betaproteobacteria were the most abundant three taxa. The high proportions of Bacteroidetes and Betaproteobacteria could be due to their generally high abundance in freshwater systems (Newton et al., 2011). The phylum Bacteroidetes is well known for its high abundance in the gut microbiota (Mahowald et al., 2009) and for having a special protein secretion system (Type IX Secretion System, T9SS) that ensures cell survival and fitness in response to its habitat (Lasica et al., 2017). It is likely that habitat (freshwatermarine) distinctness in higher taxonomic composition of DRB exists, but it is a notion that needs to be further investigated for more freshwater protist species. At lower taxonomic ranks, the DRB assemblage composition and structure along different environmental gradients and pollutant stresses showed many interesting characteristics, many of which are reported for the first time in this study, and the underlying mechanisms are discussed below.

\section{Warming Selected Specific Bacterial Species in Association With Protists}

It is interesting to observe that Pseudomonas spp., as a dominant group in $P$. bursaria, had higher relative abundances at higher temperatures (Figure 4A). It was possible that the endosymbiotic green algae Chlorella sp. became more carbon-rich at higher temperatures (Serra-Maia et al., 2016), which could supply more organic matter to the symbiotic Pseudomonas species (Yao et al., 2019). An opposite trend was observed for the dominant DRB group in the algae-free ciliate E. vannus, in which the relative abundance of Pseudoalteromonas species decreased progressively with the increase of temperature (Figure 4A). The cellular chemical composition of E. vannus could be altered with temperature, with decreasing carbon to nitrogen ratio or phosphorus ratio at higher temperatures, according to the ecological stoichiometry theory (Woods et al., 2003; $\mathrm{Fu}$ and Gong, 2017). This temperature-driven stoichiometric shift in the host could select against specific heterotrophic DRB populations, such as Pseudoalteromonas spp. The lower relative abundance of Pseudoalteromonas occurred at a higher temperature $\left(25^{\circ} \mathrm{C}\right)$, which could be attributed to the mechanism whereby Pseudoalteromonas kills other bacterial competitors, such as Vibrio, by secreting pseudoalterin, a thermolabile protease (Richards et al., 2017; Tang et al., 2020), which might lower the killing activity at a higher temperature, resulting in a higher proportion of co-occurring Vibrio in the DRB assemblage. Also, a higher temperature might enrich the type III secretion system (T3SS) of Vibrio in association with protists (Matz et al., 2011).

\section{Light-Driven DRB Assemblage in the Heterotrophic Protist, but Light-Independent DRB in the Mixotroph}

The DRB assemblage significantly responded to the light conditions, which occurred only in the heterotrophic E. vannus but not in the mixotrophic $P$. bursaria, indicating that the mixotroph could select specific bacterial populations. Interestingly, the DRB detected in the P. bursaria, i.e., the gammaproteobacterial Nevskia and the alphaproteobacterial Caulobacter and Asticcacaulis, share a common morphological character in having a stalk or holdfast at certain life stages, with which cells are able to attach onto a water-air interface or substrate surface (Stürmeyer et al., 1998; Purcell et al., 2007; Persat and Gitai, 2014). Typical species of the former two genera have also been demonstrated to be light-sensitive. For example, Nevskia ramosa often inhabit at the air-water interface and is capable of DNA reparation against UV damage (Stürmeyer et al., 1998). Visible light can act to regulate a sensory module in Caulobacter crescentus in order to increase the biochemical activity and cellular signaling for cell-surface and cell-cell attachment (Purcell et al., 2007). Asticcacaulis was detected in the microbial consortium of the oil-rich green alga Botryococcus braunii (Sambles et al., 2017). In the constant light treatment, continuous uptake of nutrients by the autotrophs, which included those in medium and the Chlorella symbionts inside the $P$. bursaria, probably lead to nutrient limitation to heterotrophic bacteria in the medium. Attachments to, or residue 
of these bacteria inside the ciliate cell may facilitate themselves to immediately "capture" the nutrients regenerated by the protist. In constant darkness, $P$. bursaria might sustain by bacterivory, and the bacteria-protist association might reduce bacterial mortality. Therefore, our study based on light manipulation and single cell analysis of protists provides evidence that mixotrophic protists are previously unrecognized hosts and micro-niches of these attaching bacteria in natural environments.

The Curvibacter phylotype occurred as DRB of the P. bursaria in all-light, all-dark, and diel-cycle treatments. Cultivation in constant darkness or at night might lower the concentration of dissolved oxygen, which provides an ecological advantage to microaerobic bacteria, such as Curvibacter sp. (Ding and Yokota, 2010). It has been demonstrated that members of this genus are able to inhibit fungal infection by interacting with other commensal bacterial species in the cnidarian Hydra (Fraune et al., 2015). In fact, at least filament fungi were recognizable via the naked eye at later periods of the cultures supplied with rice grains, indicating a risk of fungal infection of the $P$. bursaria. In this sense, it is possible for these bacterial consortiums to function in anti-fungal activity. The Variovorax phylotypes were only detected in both LL and LD treatments of the P. bursaria. This adaptive ability in such contrasting light environments is consistent with a previous study, which showed a combination of autotrophic and heterotrophic features in the genome of Variovorax paradoxus (Han et al., 2011).

The DRB in the heterotrophic ciliate E. vannus were photoperiod-dependent. Alteromonas sp. was dominant in the treatment of the diel light:dark cycles, suggesting a role of light (or darkness) in selecting these bacteria in association with the protistan cell. A similar mechanism was proposed by a previous study (Biller et al., 2018), which stated that Alteromonas may provide energy or organic compounds to cyanobacteria Prochlorococcus under the stress of darkness. The Pseudoalteromonas phylotypes were consistently present in the DRB assemblages under the conditions having a photic period, probably contributing to anti-fungal infection by more actively producing antifungal polyketide alteramides during the dark period (Moree et al., 2014). Thus, the co-occurrence of these two bacterial taxa may be of benefit to the host for a balance between rapid growth and low pathogenic infection under a natural diel light-dark condition (Liu et al., 2019).

\section{Evidence for Prevalent Antibiotic-Resistant Bacteria Sheltered in Protistan Cells}

Gomiero and Viarengo (2014) showed that the antibiotic OTC (3.2 $\mu \mathrm{M}$ to $32 \mathrm{mM}$ ) treatments induced changes in the growth, survival, endocytosis rate, and lysosomal membrane stability of the marine ciliate Euplotes crassus. Such physiological shifts might have also occurred in these two species we investigated. Apart from that, we found the dominant DRB in the OTCtreated marine ciliate E. vannus were affiliated with the members of the genus Sphingomonas and the family Alteromonadaceae (Alteromonas and Aestuariibacter) (Figure 5A), which is in line with previous findings that both Sphingomonas and Alteromonas species were resistant to antibiotics, such as OTC (Miranda and Zemelman, 2002; Dang et al., 2007). However, the relative abundance of the phylotype of the family Puniceicoccaceae (phylum Verrucomicrobia) decreased in OTC10 and was nondetectable in OTC20, indicating it is sensitive to OTC.

Both Pseudomonas and Sulfitobacter phylotypes prevailed in the DRB assemblage of mixotrophic $P$. bursaria reared in media with an antibiotic concentration up to $20 \mathrm{mg} / \mathrm{L}$, indicating high antibiotic resistance of these bacteria and that the intracellular environment may provide a favorable habitat for their survival. Indeed, it has been demonstrated that many Pseudomonas species could degrade antibiotics via efflux pumps (e.g., Jiang et al., 2014), and consortiums of these bacterial species with algae could enhance the efficiency of degrading antibiotics (Leng et al., 2020). Similarly, genes involved in aromatic compound catabolism and a type IV secretion system are present in the genome of a strain of Sulfitobacter (Ankrah et al., 2014), and Sulfitobacter in the diatom phycosphere could supply indole acetic acid to the algae in exchange for organosulfur compounds (Amin et al., 2015). Furthermore, Sulfitobacter strains isolated from marine hydrothermal vent fields were found to be antibiotic-resistant (Farias et al., 2015). All these suggest that the mixotrophic protist bearing endosymbiotic microalgae (e.g., Chlorella sp.) could play a role in accommodating these antibiotic-resistant bacteria, which represent a previously unrecognized scenario in the antibiotic resistance of environmental bacterial populations.

\section{Bacterial Co-resistance to Heavy Metals and to Protistan Digestions}

It is well known that short-rod cells of Flectobacillus form long filaments and chains of several cells, a morphology-based strategy to protect themselves from being ingested by nanoflagellates predators (Corno and Jürgens, 2006). We detected these bacteria as a member of DRB in P. bursaria, suggesting an effect of predator size in the prey-predator interactions, i.e., enlarged bacterial cell size may be effective to avoid being engulfed by small protists but not necessarily for large-celled protists, such as ciliates with wide or highly contractile cytostomes. Furthermore, the Flectobacillus phylotypes became dominant in the $\mathrm{Pb}$-treated cultures, demonstrating they are co-resistant to $\mathrm{Pb}$ and protistan digestion. However, Flectobacillus spp. were found to be a dominant bacterial group in the riverbed sediments spiked with high concentrations of metals, including $\mathrm{Cd}$ and $\mathrm{Cu}$, but not in the $\mathrm{Pb}$ and $\mathrm{Cr}$ treatments ( $\mathrm{Du}$ et al., 2018). In the Hg-treated P. bursaria culture, we also found that Flectobacillus phylotypes only accounted for a minor portion of DRB. This suggests a strain-level differentiation in the heavy metal resistance of Flectobacillus.

Acinetobacter junii is able to form biofilms on surfaces to resistant to mercury (Sarkar and Chakraborty, 2008), which is in line with our observation of its survival in P. bursaria under $\mathrm{Hg}$ stresses. In addition, our results point to the non-digestible nature of this bacterial species in ciliated protozoa, highlighting its ecological success in natural environments. Furthermore, Hydrogenophaga was also abundant in P. bursaria treated with a 
high dose of $\mathrm{Hg}$. It remains to be investigated how this hydrogenoxidizing species interacts with the host and endosymbiotic green alga within the P. bursaria under $\mathrm{Hg}$ stress, though it is known that As (III) could be oxidized by a strain of this genus under aerobic conditions (Terry et al., 2015).

Not much is known about the ecology and microbial association of Aestuariibacter, except for our recent report of this genus as a DRB in four marine and one freshwater species of ciliates (Gong et al., 2016). In the present study, we once again found this genus as a dominant group of DRB in E. vannus across all $\mathrm{Pb}^{2+}$ and $\mathrm{Cd}^{2+}$ treatments and under low-dose antibiotic treatment, indicating Aestuaribacter is a previously unrecognized superstar in co-resistance to heavy metals, antibiotics, and protistan digestion. This is probably due to their resident habitats of high-pollution stresses, such as coastal seawater and tidal flats (Yi et al., 2004; Wang et al., 2010). Pelomonas was present in the DRB of E. vannus treated with high doses of $\mathrm{Pb}^{2+}$ and $\mathrm{Cd}^{2+}$ but absent in the controls, indicating the metal tolerance of this group. Phylotypes of this genus were also detected in $\mathrm{Cd}$ - and $\mathrm{Cu}$-treated activated sludge using DGGE and sequencing (Bhat et al., 2020). Strains of Verrucomicrobia have been isolated as gut symbionts from sea cucumber, marine sponges, clamworm (Wertz et al., 2012), and the marine ciliate Euplotidium (Petroni et al., 2000). Our study showed the association between a possible new genus of the family Puniceicoccaceae with the ciliate E. vannus and for the first time their differential resistance to metals $\mathrm{Pb}$ and $\mathrm{Cd}$.

\section{CONCLUDING REMARKS}

As major players in the microbial loop and biogeochemical cycle within ecosystems, both bacteria and protists are ecologically linked not only through prey-predator food chains but also via collaboration in facing multiple stresses. Our study reveals for the first time that the assemblage composition and structure of ingested but inedible bacteria in two protists vary significantly in response to stresses of temperature, light, antibiotics, and heavy metals, indicating that the environment plays an important role in selecting specific bacterial populations in protist-bacteria associations. Our results for these two ciliate species also provide an indication that trophic mode of protists (mixotrophs vs. heterotrophs) could be a factor influencing the bacteriaprotist interactions, a notion warrants further investigation considering their increasingly recognized importance in marine food webs (Stoecker et al., 2017). Furthermore, bacterial antibiotic resistance and metal resistance have been extensively

\section{REFERENCES}

Adams, H. E., Crump, B. C., and Kling, G. W. (2010). Temperature controls on aquatic bacterial production and community dynamics in arctic lakes and streams. Environ. Microbiol. 12, 1319-1333. doi: 10.1111/j.1462-2920.2010. 02176.x

Alonso-Sáez, L., Gasol, J. M., Lefort, T., Hofer, J., and Sommaruga, R. (2006). Effect of natural sunlight on bacterial activity and differential sensitivity of natural studied in the past several decades. The ecological interactions between these resistant bacteria and protists remain poorly understudied (Nguyen et al., 2020). Our findings in this study provide evidence that there are diverse bacterial stains, which are not only resistant to antibiotics and/or heavy metals in natural environments but have also survived protistan digestion, suggesting some bacterial species/strains could use protistan cells as shelters in facing of pollution stresses.

\section{DATA AVAILABILITY STATEMENT}

The datasets presented in this study can be found in online repositories. The names of the repositories and accession numbers can be found in the article/Supplementary Material.

\section{AUTHOR CONTRIBUTIONS}

SZ did the data curation-equal, formal analysis-equal, investigation-equal, methodology-equal, resources-equal, software-equal, validation-equal, visualization-equal, and wrote the original draft-lead. QZ performed the resources-supporting and validation-supporting. XZ and CD performed the validationsupporting. JG performed the conceptualization-lead, data curation-supporting, funding acquisition-lead, methodologyequal, project administration-lead, resources-equal, supervisionequal, validation-equal, and wrote, reviewed and edited the manuscript-lead. All authors contributed to the article and approved the submitted version.

\section{FUNDING}

This work was supported by the Marine S\&T Fund of Shandong Province for Pilot National Laboratory for Marine Science and Technology (Qingdao) (No. 2018SDKJ0406-4), the Key Research Project of Frontier Science, CAS (No. QYZDB-SSW-DQC013-1), and a NSFC-CNRS Collaboration Project (No. 41311130107).

\section{SUPPLEMENTARY MATERIAL}

The Supplementary Material for this article can be found online at: https://www.frontiersin.org/articles/10.3389/fmars. 2020.00659/full\#supplementary-material

FIGURE S1 | A general scheme of research methodology.

bacterioplankton groups in northwestern mediterranean coastal waters. Appl. Environ. Microbiol. 72, 5806-5813. doi: 10.1128/AEM.00597-06

Al-Rasheid, K. A. S., and Sleigh, M. A. (1994). The effects of heavy metals on the feeding rate of Euplotes mutabilis (Tuffrau, 1960). Eur. J. Protistol. 30, 270-279. doi: 10.1016/S0932-4739(11)80073-8

Amin, S. A., Hmelo, L. R., Van Tol, H. M., Durham, B. P., Carlson, L. T., Heal, K. R., et al. (2015). Interaction and signalling between a cosmopolitan phytoplankton and associated bacteria. Nature 522, 98-101. doi: 10.1038/nature14488 
Ankrah, N. Y. D., Lane, T., Budinoff, C. R., Hadden, M. K., and Buchan, A. (2014). Draft genome sequence of Sulfitobacter sp. CB2047, a member of the Roseobacter clade of marine bacteria, isolated from an Emiliania huxleyi bloom. Genome Announc. 2:e01125-14. doi: 10.1128/genomeA.01125-14

Ashelford, K. E., Chuzhanova, N. A., Fry, J. C., Jones, A. J., and Weightman, A. J. (2006). New screening software shows that most recent large 16S rRNA gene clone libraries contain chimeras. Appl. Environ. Microbiol. 72, 5734-5741. doi: 10.1128/AEM.00556-06

Azam, F., Fenchel, T., Field, J. G., Gray, J. S., Meyer-Reil, L. A., and Thingstad, F. (1983). The ecological role of water-column microbes in the sea. Mar. Ecol. Prog. Ser. 10, 257-263. doi: 10.3354/meps010257

Bhat, S. A., Cui, G., Li, W., Wei, Y., and Li, F. (2020). Effect of heavy metals on the performance and bacterial profiles of activated sludge in a semi-continuous reactor. Chemosphere 241:125035. doi: 10.1016/j.chemosphere.2019.125035

Biller, S. J., Coe, A., Roggensack, S. E., and Chisholm, S. W. (2018). Heterotroph interactions alter Prochlorococcus transcriptome dynamics during extended periods of darkness. mSystems 3:e0040-18. doi: 10.1128/mSystems.00040- 18

Boenigk, J., Matz, C., Jürgens, K., and Arndt, H. (2001). The influence of preculture conditions and food quality on the ingestion and digestion process of three species of heterotrophic nanoflagellates. Microb. Ecol. 42, 168-176. doi: 10.1007/ s002480000116

Corno, G., and Jürgens, K. (2006). Direct and indirect effects of protist predation on population size structure of a bacterial strain with high phenotypic plasticity. Appl. Environ. Microbiol. 72, 78-86. doi: 10.1128/AEM.72.1.78-86.2006

Dang, H., Zhang, X., Song, L., Chang, Y., and Yang, G. (2007). Molecular determination of oxytetracycline-resistant bacteria and their resistance genes from mariculture environments of China. J. Appl. Microbiol. 103, 2580-2592. doi: $10.1111 / j .1365-2672.2007 .03494 . x$

Darriba, D., Taboada, G. L., Doallo, R., and Posada, D. (2012). jModelTest 2: more models, new heuristics and parallel computing. Nat. Methods 9, 772-772. doi: 10.1038/nmeth.2109

Ding, L., and Yokota, A. (2010). Curvibacter fontana sp. nov., a microaerobic bacteria isolated from well water. J. Gen. Appl. Microbiol. 56, 267-271. doi: 10.2323/jgam.56.267

Du, H., Harata, N., and Li, F. (2018). Responses of riverbed sediment bacteria to heavy metals: integrated evaluation based on bacterial density, activity and community structure under well-controlled sequencing batch incubation conditions. Water Res. 130, 115-126. doi: 10.1016/j.watres.2017.10.070

Farias, P., Espírito Santo, C., Branco, R., Francisco, R., Santos, S., Hansen, L., et al. (2015). Natural hot spots for gain of multiple resistances: arsenic and antibiotic resistances in heterotrophic, aerobic bacteria from marine hydrothermal vent fields. Appl. Environ. Microbiol. 81, 2534-2543. doi: 10.1128/AEM.03240- 14

Farnelid, H. M., Turk-Kubo, K. A., and Zehr, J. P. (2016). Identification of associations between bacterioplankton and photosynthetic picoeukaryotes in coastal waters. Front. Microbiol. 7:339. doi: 10.3389/fmicb.2016.00339

First, M. R., Park, N. Y., Berrang, M. E., Meinersmann, R. J., Bernhard, J. M., Gast, R. J., et al. (2012). Ciliate ingestion and digestion: flow cytometric measurements and regrowth of a digestion-resistant Campylobacter jejuni. J. Eukaryot. Microbiol. 59, 12-19. doi: 10.1111/j.1550-7408.2011.00589.x

Fraune, S., Anton-Erxleben, F., Augustin, R., Franzenburg, S., Knop, M., Schröder, K., et al. (2015). Bacteria-bacteria interactions within the microbiota of the ancestral metazoan Hydra contribute to fungal resistance. ISME J. 9, 1543-1556. doi: 10.1038/ismej.2014.239

Fu, R., and Gong, J. (2017). Single cell analysis linking ribosomal (r)DNA and rRNA copy numbers to cell size and growth rate provides insights into molecular protistan ecology. J. Eukaryot. Microbiol. 64, 885-896. doi: 10.1111/jeu.12425

Galtier, N., Gouy, M., and Gautier, C. (1996). SEAVIEW and PHYLO_WIN: two graphic tools for sequence alignment and molecular phylogeny. Bioinformatics 12, 543-548. doi: 10.1093/bioinformatics/12.6.543

Gomiero, A., and Viarengo, A. (2014). Effects of elevated temperature on the toxicity of copper and oxytetracycline in the marine model, Euplotes crassus: a climate change perspective. Environ. Pollut. 194, 262-271. doi: 10.1016/j. envpol.2014.07.035

Gong, J., Qing, Y., Guo, X., and Warren, A. (2014). "Candidatus Sonnebornia yantaiensis", a member of candidate division OD1, as intracellular bacteria of the ciliated protist Paramecium bursaria (Ciliophora. Oligohymenophorea). Syst. Appl. Microbiol. 37, 35-41. doi: 10.1016/j.syapm.2013.08.007
Gong, J., Qing, Y., Zou, S., Fu, R., Su, L., Zhang, X., et al. (2016). Protist-bacteria associations: gammaproteobacteria and alphaproteobacteria are prevalent as digestion-resistant bacteria in ciliated protozoa. Front. Microbiol. 7:498. doi: 10.3389/fmicb.2016.00498

Guindon, S., Delsuc, F., Dufayard, J.-F., and Gascuel, O. (2009). "Estimating maximum likelihood phylogenies with PhyML," in Bioinformatics for DNA Sequence Analysis. Methods in Molecular Biology, ed. D. Posada (Totowa, NJ: Humana Press), 113-137. doi: 10.1007/978-1-59745-251-9_6

Han, J.-I., Choi, H.-K., Lee, S.-W., Orwin, P. M., Kim, J., Laroe, S. L., et al. (2011). Complete genome sequence of the metabolically versatile plant growthpromoting endophyte Variovorax paradoxus S110. J. Bacteriol. 193, 1183-1190. doi: 10.1128/JB.00925-10

Hu, A., Yang, X., Chen, N., Hou, L., Ma, Y., and Yu, C.-P. (2014). Response of bacterial communities to environmental changes in a mesoscale subtropical watershed, Southeast China. Sci. Total Environ. 472, 746-756. doi: 10.1016/j. scitotenv.2013.11.097

Huber, T., Faulkner, G., and Hugenholtz, P. (2004). Bellerophon: a program to detect chimeric sequences in multiple sequence alignments. Bioinformatics 20 , 2317-2319. doi: 10.1093/bioinformatics/bth226

Ibekwe, A. M., Ma, J., and Murinda, S. E. (2016). Bacterial community composition and structure in an urban river impacted by different pollutant sources. Sci. Total Environ. 566-567, 1176-1185. doi: 10.1016/j.scitotenv.2016.05.168

Izaguirre, I., Sinistro, R., Schiaffino, M. R., Sánchez, M. L., Unrein, F., and Massana, R. (2012). Grazing rates of protists in wetlands under contrasting light conditions due to floating plants. Aquat. Microb. Ecol. 65, 221-232. doi: 10.3354/ame01547

Jiang, B., Li, A., Cui, D., Cai, R., Ma, F., and Wang, Y. (2014). Biodegradation and metabolic pathway of sulfamethoxazole by Pseudomonas psychrophila HA4, a newly isolated cold-adapted sulfamethoxazole-degrading bacterium. Appl. Microbiol. Biotechnol. 98, 4671-4681. doi: 10.1007/s00253-013-5488-3

Jousset, A. (2012). Ecological and evolutive implications of bacterial defences against predators. Environ. Microbiol. 14, 1830-1843. doi: 10.1111/j.1462-2920. 2011.02627.x

Katoh, K., and Standley, D. M. (2013). MAFFT multiple sequence alignment software version 7: improvements in performance and usability. Mol. Biol. Evol. 30, 772-780. doi: 10.1093/molbev/mst010

Kim, S.-H., Jung, M.-Y., and Lee, Y.-M. (2011). Effect of heavy metals on the antioxidant enzymes in the marine ciliate Euplotes crassus. Toxicol. Environ. Health Sci. 3, 213-219. doi: 10.1007/s13530-011-0103-4

Kodama, Y., and Fujishima, M. (2014). Symbiotic Chlorella variabilis incubated under constant dark conditions for 24 hours loses the ability to avoid digestion by host lysosomal enzymes in digestive vacuoles of host ciliate Paramecium bursaria. FEMS Microbiol. Ecol. 90, 946-955. doi: 10.1111/1574-6941.12448

Lane, D. J. (1991). 16S/23S rRNA Sequencing. New York, NY: John Wiley \& Sons Ltd.

Lasica, A. M., Ksiazek, M., Madej, M., and Potempa, J. (2017). The type IX secretion system (T9SS): highlights and recent insights into its structure and function. Front. Cell. Infect. Microbiol. 7:215. doi: 10.3389/fcimb.2017.00215

Leng, L., Wei, L., Xiong, Q., Xu, S., Li, W., Lv, S., et al. (2020). Use of microalgae based technology for the removal of antibiotics from wastewater: a review. Chemosphere 238:124680. doi: 10.1016/j.chemosphere.2019.124680

Liu, J., Meng, Z., Liu, X., and Zhang, X.-H. (2019). Microbial assembly, interaction, functioning, activity and diversification: a review derived from community compositional data. Mar. Life Sci. Technol. 1, 112-128. doi: 10.1007/s42995019-00004-3

Mahowald, M. A., Rey, F. E., Seedorf, H., Turnbaugh, P. J., Fulton, R. S., Wollam, A., et al. (2009). Characterizing a model human gut microbiota composed of members of its two dominant bacterial phyla. Proc. Natl. Acad. Sci. U.S.A. 106, 5859-5864. doi: 10.1073/pnas.0901529106

Martinez-Garcia, M., Brazel, D., Poulton, N. J., Swan, B. K., Gomez, M. L., Masland, D., et al. (2012). Unveiling in situ interactions between marine protists and bacteria through single cell sequencing. ISME J. 6, 703-707. doi: 10.1038/ismej. 2011.126

Matz, C., and Kjelleberg, S. (2005). Off the hook-how bacteria survive protozoan grazing. Trends Microbiol. 13, 302-307. doi: 10.1016/j.tim.2005.05.009

Matz, C., Nouri, B., Mccarter, L., and Martinez-Urtaza, J. (2011). Acquired type III secretion system determines environmental fitness of epidemic Vibrio 
parahaemolyticus in the interaction with bacterivorous protists. PLoS One 6:e20275. doi: 10.1371/journal.pone.0020275

Miranda, C. D., and Zemelman, R. (2002). Bacterial resistance to oxytetracycline in Chilean salmon farming. Aquaculture 212, 31-47. doi: 10.1016/S0044-8486(02) 00124-2

Montagnes, D. J. S., and Weisse, T. (2000). Fluctuating temperatures affect growth and production rates of planktonic ciliates. Aquat. Microb. Ecol. 21, 97-102. doi: $10.3354 /$ ame 021097

Moree, W. J., Mcconnell, O. J., Nguyen, D. D., Sanchez, L. M., Yang, Y.-L., Zhao, X., et al. (2014). Microbiota of healthy corals are active against fungi in a lightdependent manner. ACS Chem. Biol. 9, 2300-2308. doi: 10.1021/cb500432j

Newton, R. J., Jones, S. E., Eiler, A., Mcmahon, K. D., and Bertilsson, S. (2011). A guide to the natural history of freshwater lake bacteria. Microbiol. Mol. Biol. Rev. 75, 14-49. doi: 10.1128/MMBR.00028-10

Nguyen, B.-A. T., Chen, Q.-L., He, J.-Z., and Hu, H.-W. (2020). Microbial regulation of natural antibiotic resistance: understanding the protist-bacteria interactions for evolution of soil resistome. Sci. Total Environ. 705:135882. doi: 10.1016/j.scitotenv.2019.135882

Osborn, A. M., Moore, E. R. B., and Timmis, K. N. (2000). An evaluation of terminal-restriction fragment length polymorphism (T-RFLP) analysis for the study of microbial community structure and dynamics. Environ. Microbiol. 2, 39-50. doi: 10.1046/j.1462-2920.2000.00081.x

Pernthaler, J. (2005). Predation on prokaryotes in the water column and its ecological implications. Nat. Rev. Microbiol. 3, 537-546. doi: 10.1038/ nrmicro1180

Persat, A., and Gitai, Z. (2014). Bacterial evolution: rewiring modules to get in shape. Curr. Biol. 24, R522-R524. doi: 10.1016/j.cub.2014.04.022

Petroni, G., Spring, S., Schleifer, K.-H., Verni, F., and Rosati, G. (2000). Defensive extrusive ectosymbionts of Euplotidium (Ciliophora) that contain microtubulelike structures are bacteria related to Verrucomicrobia. Proc. Natl. Acad. Sci. U.S.A. 97, 1813-1817. doi: 10.1073/pnas.030438197

Pucciarelli, S., Devaraj, R. R., Mancini, A., Ballarini, P., Castelli, M., Schrallhammer, M., et al. (2015). Microbial consortium associated with the antarctic marine ciliate Euplotes focardii: an investigation from genomic sequences. Microb. Ecol. 70, 484-497. doi: 10.1007/s00248-015-0568-9

Purcell, E. B., Siegal-Gaskins, D., Rawling, D. C., Fiebig, A., and Crosson, S. (2007). A photosensory two-component system regulates bacterial cell attachment. Proc. Natl. Acad. Sci. U.S.A. 104, 18241-18246. doi: 10.1073/pnas.0705887104

Richards, G. P., Watson, M. A., Needleman, D. S., Uknalis, J., Boyd, E. F., and Fay, J. P. (2017). Mechanisms for Pseudoalteromonas piscicida-induced killing of vibrios and other bacterial pathogens. Appl. Environ. Microbiol. 83:e00175-17. doi: 10.1128/AEM.00175-17

Ronquist, F., and Huelsenbeck, J. P. (2003). MrBayes 3: Bayesian phylogenetic inference under mixed models. Bioinformatics 19, 1572-1574. doi: 10.1093/ bioinformatics/btg180

Sambles, C., Moore, K., Lux, T. M., Jones, K., Littlejohn, G. R., Gouveia, J. D., et al. (2017). Metagenomic analysis of the complex microbial consortium associated with cultures of the oil-rich alga Botryococcus braunii. MicrobiologyOpen 6:e00482. doi: 10.1002/mbo3.482

Sarkar, S., and Chakraborty, R. (2008). Quorum sensing in metal tolerance of Acinetobacter junii BB1A is associated with biofilm production. FEMS Microbiol. Lett. 282, 160-165. doi: 10.1111/j.1574-6968.2008.01080.x

Schloss, P. D., Westcott, S. L., Ryabin, T., Hall, J. R., Hartmann, M., Hollister, E. B., et al. (2009). Introducing mothur: open-source, platform-independent, community-supported software for describing and comparing microbial communities. Appl. Environ. Microbiol. 75, 7537-7541. doi: 10.1128/AEM. 01541-09

Serra-Maia, R., Bernard, O., Gonçalves, A., Bensalem, S., and Lopes, F. (2016). Influence of temperature on Chlorella vulgaris growth and mortality rates in a photobioreactor. Algal Res. 18, 352-359. doi: 10.1016/j.algal.2016.06.016

Sherr, B. F., Sherr, E. B., and Rassoulzadegan, F. (1988). Rates of digestion of bacteria by marine phagotrophic protozoa: temperature dependence. Appl. Environ. Microbiol. 54, 1091-1095. doi: 10.1128/aem.54.5.1091-1095.1988

Shibata, A., Takahashi, F., Kasahara, M., and Imamura, N. (2016). Induction of maltose release by light in the endosymbiont Chlorella variabilis of Paramecium bursaria. Protist 167, 468-478. doi: 10.1016/j.protis.2016.08.007
Šimek, K., Kasalický, V., Jezbera, J., Horňák, K., Nedoma, J., Hahn, M. W., et al. (2013). Differential freshwater flagellate community response to bacterial food quality with a focus on Limnohabitans bacteria. ISME J. 7, 1519-1530. doi: 10.1038/ismej.2013.57

Stoecker, D. K., Hansen, P. J., Caron, D. A., and Mitra, A. (2017). Mixotrophy in the marine plankton. Ann. Rev. Mar. Sci. 9, 311-335. doi: 10.1146/annurev-marine010816-060617

Storesund, J. E., Erga, S. R., Ray, J. L., Thingstad, T. F., and Sandaa, R.-A. (2015). Top-down and bottom-up control on bacterial diversity in a western Norwegian deep-silled fjord. FEMS Microbiol. Ecol. 91:fiv076. doi: 10.1093/femsec/fiv076

Stürmeyer, H., Overmann, J., Babenzien, H.-D., and Cypionka, H. (1998). Ecophysiological and phylogenetic studies of Nevskia ramosa in pure culture. Appl. Environ. Microbiol. 64, 1890-1894. doi: 10.1128/aem.64.5.1890-1894. 1998

Tang, B.-L., Yang, J., Chen, X.-L., Wang, P., Zhao, H.-L., Su, H.-N., et al. (2020). A predator-prey interaction between a marine Pseudoalteromonas sp. and Gram-positive bacteria. Nat. Commun. 11:285. doi: 10.1038/s41467-01914133-x

Terry, L. R., Kulp, T. R., Wiatrowski, H., Miller, L. G., and Oremland, R. S. (2015). Microbiological oxidation of antimony (III) with oxygen or nitrate by bacteria isolated from contaminated mine sediments. Appl. Environ. Microbiol. 81, 8478-8488. doi: 10.1128/AEM.01970-15

Wang, Q., Garrity, G. M., Tiedje, J. M., and Cole, J. R. (2007). Naïve bayesian classifier for rapid assignment of rRNA sequences into the new bacterial taxonomy. Appl. Environ. Microbiol. 73, 5261-5267. doi: 10.1128/AEM.0006207

Wang, Y., Wang, H., Liu, J., Lai, Q., Shao, Z., Austin, B., et al. (2010). Aestuariibacter aggregatus sp. nov., a moderately Halophilic bacterium isolated from seawater of the Yellow Sea. FEMS Microbiol. Lett. 309, 48-54. doi: 10.1111/ j.1574-6968.2010.02011.x

Wertz, J. T., Kim, E., Breznak, J. A., Schmidt, T. M., and Rodrigues, J. L. M. (2012). Genomic and physiological characterization of the Verrucomicrobia isolate Diplosphaera colitermitum gen. nov., sp. nov., reveals microaerophily and nitrogen fixation genes. Appl. Environ. Microbiol. 78, 1544-1555. doi: 10. 1128/AEM.06466-11

Woods, H. A., Makino, W., Cotner, J. B., Hobbie, S. E., Harrison, J. F., Acharya, K., et al. (2003). Temperature and the chemical composition of poikilothermic organisms. Funct. Ecol. 17, 237-245. doi: 10.1046/j.1365-2435.2003. 00724.x

Xu, Y., Shen, Z., Gentekaki, E., Xu, J., and Yi, Z. (2020). Comparative transcriptome analyses during the vegetative cell cycle in the mono-cellular organism Pseudokeronopsis erythrina (Alveolata, Ciliophora). Microorganisms 8:108. doi: 10.3390/microorganisms8010108

Yao, S., Lyu, S., An, Y., Lu, J., Gjermansen, C., and Schramm, A. (2019). Microalgaebacteria symbiosis in microalgal growth and biofuel production: a review. J. Appl. Microbiol. 126, 359-368. doi: 10.1111/jam.14095

Yi, H., Bae, K. S., and Chun, J. (2004). Aestuariibacter salexigens gen. nov., sp. nov. and Aestuariibacter halophilus sp. nov., isolated from tidal flat sediment, and emended description of Alteromonas macleodii. Int. J. Syst. Evol. Microbiol. 54, 571-576. doi: 10.1099/ijs.0.02798-0

Zou, S., Zhang, Q., and Gong, J. (2020). Comparative transcriptomics reveals distinct gene expressions of a model ciliated protozoan feeding on bacteriafree medium, digestible, and digestion-resistant bacteria. Microorganisms 8:559. doi: 10.3390/microorganisms8040559

Conflict of Interest: The authors declare that the research was conducted in the absence of any commercial or financial relationships that could be construed as a potential conflict of interest.

Copyright (c) 2020 Zou, Zhang, Zhang, Dupuy and Gong. This is an open-access article distributed under the terms of the Creative Commons Attribution License (CC BY). The use, distribution or reproduction in other forums is permitted, provided the original author(s) and the copyright owner(s) are credited and that the original publication in this journal is cited, in accordance with accepted academic practice. No use, distribution or reproduction is permitted which does not comply with these terms. 